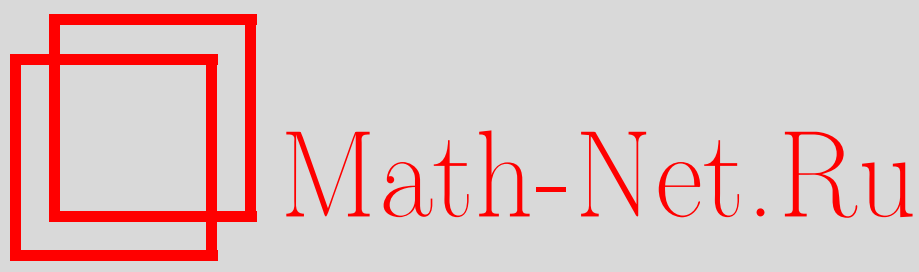

Ф. Х. Мукминов, И. М. Биккулов, О стабилизации нормы решения одной смешанной задачи для параболических уравнений 4-го и 6-го порядков в неограниченной области, Матем. сб., 2004, том 195, номер 3, 115-142

DOI: https://doi.org/10.4213/sm810

Использование Общероссийского математического портала Math-Net.Ru подразумевает, что вы прочитали и согласны с пользовательским соглашением http://www.mathnet.ru/rus/agreement

Параметры загрузки:

IP: 3.85 .73 .92

26 апреля 2023 г., 02:44:31 


\section{О стабилизации нормы решения одной смешанной задачи для параболических уравнений 4-го и 6-го порядков в неограниченной области}

\footnotetext{
В работе исследуется поведение при $t \rightarrow \infty$ решения смешанной задачи для параболических уравнений в неограниченной области с двумя выходами на бесконечность. Выделен класс областей, для которых установлена определяемая геометрией области оценка, характеризующая стабилизацию решения. Доказано, что эта оценка является, в определенном смысле, точной в широком классе областей с двумя выходами на бесконечность.

Библиограффия: 16 названий.
}

Пусть $\Omega$ - произвольная неограниченная область $n$-мерного пространства $\mathbb{R}_{n}$, $n \geqslant 2, x=\left(x_{1}, \ldots, x_{n}\right)$ - точка $\mathbb{R}_{n}$.

Рассмотрим для параболического уравнения

$$
u_{t}=-L^{2} u, \quad L u \equiv \sum_{i, j=1}^{n}\left(a_{i j}(x) u_{x_{i}}\right)_{x_{j}},
$$

в цилиндрической области $D=\{t>0\} \times \Omega$ следующую смешанную задачу

$$
\begin{gathered}
\left.u(t, x)\right|_{x \in \partial \Omega}=\left.L u(t, x)\right|_{x \in \partial \Omega}=0, \\
u(0, x)=\varphi(x), \quad \varphi \in L_{2}(\Omega),
\end{gathered}
$$

где начальная функция $\varphi(x)$ имеет ограниченный носитель

$$
\varphi(x)=0 \text { при }|x|>R_{0} .
$$

Коэффициенты уравнения - измеримые функции, удовлетворяюшие для почти всех $(t, x) \in D$ неравенствам

$$
\begin{gathered}
\nu|\eta|^{2} \leqslant \sum_{i, j=1}^{n} a_{i j}(x) \eta_{i} \eta_{j} \leqslant \mu|\eta|^{2} \\
\forall \eta=\left(\eta_{1}, \ldots, \eta_{n}\right) \in \mathbb{R}_{n}, \quad|\eta|^{2}=\eta_{1}^{2}+\cdots+\eta_{n}^{2}, \\
\left|\sum_{i, j=1}^{n}\left(a_{i j}(x)\right)_{x_{j}}\right| \leqslant g(|x|), \quad|x|>R_{0},
\end{gathered}
$$

где $\nu, \mu$ - положительные действительные числа. Ограничения на функцию $g(r)$ определим позже.

Работа вьполнена при поддержке Российского фонда фундаментальных исследований (грант № 99-01-00934). 
Настояшая работа посвящена исследованию поведения при больших значениях времени $L_{2}(\Omega)$-нормы решения $u(t, x)$ задачи $(1)-(3)$.

Известно много работ, в которых изучаются вопросы, в определенном смысле близкие к рассматриваемой задаче (см. [1]-[4]). Они посвящены в основном параболическим уравнениям второго порядка, для которых известен принцип максимума и ряд других свойств решения, позволяюших установить точные оценки его поведения при больших значениях времени. В случае же смешанных задач для параболических уравнений высокого порядка имеются оценки решения сверху, но нам не известны результаты, каким-либо образом подтверждающие их точность.

Направление исследований, в русле которого лежит и настоящая работа, было заложено в работах А. К. Гушина [1], [2], посвященных изучению стабилизации решений второй смешанной задачи для параболического уравнения второго порядка в неограниченной области. В частности, им получена следующая оценка решения. Пусть $v(r)$ - объем пересечения $\Omega(r)=K(r) \cap \Omega, K(r)=\{|x|<r\}$. Тогда для широкого класса областей $\Omega$, удовлетворяюших некоторым условиям изопериметрического типа, решение $u(t, x)$ второй смешанной задачи удовлетворяет неравенству

$$
|u(t, x)| \leqslant \frac{\Gamma_{0}\|\varphi\|_{L_{1}(\Omega)}}{v(\sqrt{t})} .
$$

Доказана также точность этой оценки. Поведение при $t \rightarrow \infty$ решений смешанной задачи в нецилиндрической области (с граничным условием, обеспечивающим сохранение энергии) исследовалось В. И. Ушаковым в [5]. Подробный обзор работ по стабилизации решений параболических уравнений второго порядка можно найти в работах [3], [6], [7].

Для параболического уравнения высокого порядка

$$
u_{t}+(-1)^{m} \sum_{|\alpha|=m} D^{\alpha} A_{\alpha}\left(t, x, u, D u, \ldots, D^{m} u\right)=0, \quad m \geqslant 1,
$$

в цилиндрической области $D$ с граничными и начальньми условиями

$$
\begin{gathered}
\left.D^{\beta} u\right|_{\partial \Omega \times\{t>0\}}=0, \quad|\beta| \leqslant m-1, \\
u(0, x)=\varphi(x), \quad x \in \Omega,
\end{gathered}
$$

где $A_{\alpha}(t, x, \xi)$ - каратеодоровы функции, удовлетворяюшие условиям

$$
\begin{gathered}
\sum_{|\alpha|=m} A_{\alpha}(t, x, \xi) \xi_{\alpha}^{m} \geqslant c_{0} \sum_{|\alpha|=m}\left|\xi_{\alpha}^{m}\right|^{2}, \\
\sum_{|\alpha|=m}\left|A_{\alpha}(t, x, \xi)\right| \geqslant c_{1} \sum_{|\alpha|=m}\left|\xi_{\alpha}^{m}\right|
\end{gathered}
$$

для любого вектора $\xi=\left(\xi^{0}, \xi^{1}, \ldots, \xi^{m}\right), \xi^{i}=\left(\xi_{\alpha}^{i}\right),|\alpha|=i, c_{0}, c_{1}$-положительные постоянные, А. $\Phi$. Тедеевым [8] получен следуюший результат. Пусть $\lambda(r), r>0,-$ первое собственное число оператора Лапласа в области $\Omega(r)$ с условием Дирихле 
на границе и $r(t), t>0,-$ обратная к $F(r)=r /[\lambda(r)]^{(2 m-1) / 2}$ функция. Предполагается, что $\Omega$ удовлетворяет условиям: $\lim _{r \rightarrow \infty} r^{2} \lambda(r)=\infty, \lim _{r \rightarrow \infty} \lambda(r)=0$. Тогда при условии (4) для достаточно больших $t>T_{1}^{*}$ в [8] установлено неравенство

$$
\int_{\Omega} u^{2}(t, x) d x \leqslant \Gamma_{1} \exp \left(-\gamma_{0}\left(\frac{r^{2 m}(t)}{t}\right)^{1 /(2 m-1)}\right)
$$

где $\Gamma_{1}, \gamma_{0}$ - положительные постоянные. В частности, для трубчатых областей вида

$$
\Omega[f]=\left\{x \in \mathbb{R}_{n}, x=\left(x_{1}, x^{\prime}\right):\left|x^{\prime}\right|<f\left(x_{1}\right), x_{1}>0\right\}
$$

с функцией $f\left(x_{1}\right)=x_{1}^{\alpha}, \alpha \in(0,1)$, приведенная оценка примет вид:

$$
\int_{\Omega} u^{2}(t, x) d x \leqslant \Gamma_{2} \exp \left(-\gamma_{1} t^{(1-\alpha) /(1+\alpha(2 m-1))}\right), \quad t>T_{2}^{*} .
$$

Указанные оценки совпадают с соответствующими оценками для решения первой смешанной задачи для линейного параболического уравнения высокого порядка, полученными ранее в работе [9]. Отметим, что в работе [10] на основе принципа максимума при $m=1$, т.е. для линейного параболического уравнения второго порядка, получена равномерная оценка для $x \in \Omega$

$$
|u(t, x)| \leqslant \Gamma_{3} \exp \left(-\gamma_{2} \frac{r^{2}(t)}{t}\right)\|\varphi\|_{L_{2}(\Omega)}, \quad t>T_{3}^{*},
$$

и доказано, что она является точной по порядку стремления к нулю при $t \rightarrow \infty$, в частности, для трубчатых областей вида $\Omega\left[x_{1}^{\alpha}\right]$.

Для простоты ограничимся рассмотрением области $\Omega$, имеющей два выхода на бесконечность, расположенных вдоль оси $O x_{1}$, т.е.

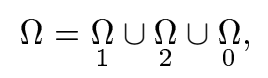

где $\underset{0}{\Omega}$ - ограниченная область, области $\Omega_{i}, i=1,2$, расположены в полуплоскостях $\left\{(-1)^{i} x_{1}>0\right\}$ соответственно. Предполагается, что области $\underset{i}{\Omega^{r}}=\{x \in \underset{i}{\Omega}$ : $\left.(-1)^{i} x_{1}<r\right\}$ ограничены при $r>0$. При этом можно $\underset{0}{\Omega}$ выбрать так, чтобы пересечение $\underset{0}{\Omega} \cap \partial \Omega$ было достаточно “весомым" (см. ниже неравенство (13)). Введем обозначение $\Omega_{i}^{b}=\left\{x \in \underset{i}{a}: a<(-1)^{i} x_{1}<b\right\}$, при этом значения индексов $a=0, b=\infty$ могут опускаться.

Для рассматриваемой задачи (1)-(3) мы получаем равномерную оценку вида (8) в том смысле, что она справедлива не только для решения $u$ задачи (1)-(3) в области $\Omega$, но и для решения $u_{r}$ задачи (1)-(3) в области $\Omega_{i}^{r}, i=1,2, r \geqslant R_{0}$, с постоянньми, не зависящими от $r$. Далее, мы подтверждаем точность нижеследуюшей оценки (17), выбирая для каждого момента времени $t>T$ такую область $\Omega_{i}^{r(t)}$, решение в которой удовлетворяет оценке

$$
\Gamma_{3} \exp \left(-\gamma_{3}\left(\frac{r^{4}(t)}{t}\right)^{1 / 3}\right) \leqslant \int_{\substack{\Omega_{i}^{r(t)} \\ r(t)}} u_{r(t, x)}^{2} d x .
$$


Определим функции $\lambda_{i}(r), i=1,2, r>0$, следующим образом:

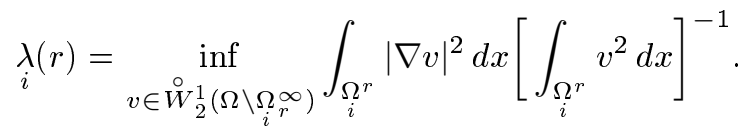

Очевидно, $\underset{i}{\lambda}(r), r>0,-$ невозрастаюшие функции.

Всюду в работе предполагается, что граница области $\Omega$ принадлежит равномерно классу $C^{2}$, в оговоренных случаях равномерно классу $C^{3}$, а также

$$
\begin{gathered}
\lim _{r \rightarrow \infty} r_{i}^{2} \lambda(r)=\infty, \\
\lim _{r \rightarrow \infty} \lambda_{i}^{\lambda}(r)=0, \quad i=1,2 .
\end{gathered}
$$

Потребуем, чтобы для области $\Omega$ выполнялось неравенство

$$
\mu^{*}=\inf _{v \in C_{0}^{\infty}(\Omega)} \int_{\substack{\Omega \\ 0}}|\nabla v|^{2} d x\left[\int_{\substack{\Omega \\ 0}} v^{2} d x\right]^{-1}>0 .
$$

Это ограничение несущественно, поскольку имеется произвол в выборе области $\Omega$. Нетрудно доказать, что оно выполнено, если пересечение $\underset{0}{\Omega} \cap \partial \Omega$ имеет ненулевую меру размерности $(n-1)$. Кроме этого потребуем, чтобы функция $g(r)$ удовлетворяла при достаточно больших значениях $r \geqslant R_{1}$ неравенству

$$
g(r) \leqslant V \min _{i}\left\{\lambda_{i}^{1 / 2}(r)\right\}
$$

с определенной в п. 2 постоянной $V$, зависящей только от $n, \nu, \mu$ и $R_{0}$. Рассмотрим монотонно возрастающие непрерьвные функции $F_{i}(r)=r[\underset{i}{\lambda}(r)]^{-3 / 2}, r>0$, $i=1,2$. Обозначим через $r_{i}(t), t>0$, обратные функции к $F_{i}(r)$ соответственно. Отметим, что так как $t=r_{i}(t)\left[\lambda_{i}\left(r_{i}(t)\right)\right]^{-3 / 2}$, то справедливы равенства

$$
\left[\frac{r_{i}^{4}(t)}{t}\right]^{1 / 3}=t \underset{i}{\lambda^{2}}\left(r_{i}(t)\right)=r_{i}(t) \underset{i}{\lambda_{i}^{1 / 2}}\left(r_{i}(t)\right)
$$

В следуюшей теореме $u(t, x)$ - решение задачи (1)-(3) в области $\Omega$, a $_{i} u_{r}(t, x)$, $i=1,2,-$ решения той же задачи в областях $\Omega_{i}^{r}$ соответственно.

ТЕОРема 1. Пусть область $\Omega$ удовлетворяет условиям (11), (12), коэффициенты уравнения (1) удовлетворяют условиям (5), (6) и выполнено условие (14). Тогда найдутся такое положстельное число ћ, зависящее только от $n, \mu$ и $\nu$, и такие числа $T_{1}, M_{1}$, зависящие от $n, \nu, \mu, R_{1} u R_{0}$ $\left(\operatorname{supp} \varphi \subset K\left(R_{0}\right)\right)$, что для всех $t \geqslant T_{1}$ справедливо неравенство

$$
\int_{\Omega} u^{2}(t, x) d x \leqslant M_{1} \exp \left(-\varkappa_{1} \min _{i}\left[\frac{r_{i}^{4}(t)}{t}\right]^{1 / 3}\right)(1+t)\|\varphi\|_{L_{2}(\Omega)}^{2} .
$$


Такая же оценка справедлива и для функций $u_{i}(t, x)$ :

$$
\max _{i} \int_{\Omega_{i}^{r}} u_{i}^{2}(t, x) d x \leqslant M_{1} \exp \left(-\varkappa_{1} \min _{i}\left[\frac{r_{i}^{4}(t)}{t}\right]^{1 / 3}\right)(1+t)\|\varphi\|_{L_{2}(\Omega)}^{2} \quad \forall r>2 R_{0}
$$

с теми же самыми постоянными $M_{1} u \varkappa_{1}$.

Отметим, что из (15) и (11) следует соотношение

$$
\lim _{t \rightarrow \infty} \min _{i}\left[\frac{r_{i}^{4}(t)}{t}\right]=\infty
$$

т.е. экспонента в (16) стремится к нулю при $t \rightarrow \infty$. В частности, для областей вида $\Omega\left[x_{1}^{\alpha}\right]$ оценка (16) примет вид

$$
\int_{\Omega} u^{2}(t, x) d x \leqslant M_{1} \exp \left(-\varkappa_{1} t^{(1-\alpha) /(1+3 \alpha)}\right), \quad t>T_{4}^{*} .
$$

Оценку снизу установим для областей с двумя выходами на бесконечность следующего вида. Если выбрать ось $O x_{1}$ направленной вдоль оси вращения области $\Omega$, то каждый рукав имеет вид $\Omega=\Omega\left[f_{i}\right], i=1,2$. Предполагается, что функции $f_{i}, i=1,2$, принадлежат классу $C^{2}[0, \infty)$. Будем говорить, что область $\Omega[f]$ удовлетворяет условиям А), В) или С), если соответственно:

А) существуют положительные постоянные $Q, P$ такие, что для произвольной точки $z=\left(s, 0^{\prime}\right) \in O x_{1}, s>P$, выполнено неравенство

$$
Q \int_{s-\rho(s) / 2}^{s+\rho(s) / 2} \frac{d r}{f(r)} \geqslant 1
$$

где $\rho(s)$ - радиусы наибольших шаров $B(\rho, z)$ с центром в $z$, лежащих в $\Omega[f]$;

В) сушествует положительная постоянная $C$ такая, что

$$
\int_{P}^{r} \frac{d \rho}{f(\rho)} \leqslant C \frac{r}{f(r)}, \quad r \geqslant P
$$

C) существует положительное число $\sigma$ такое, что

$$
f(r) \geqslant \sigma \rho_{m}(r), \quad r \geqslant P,
$$

где $\rho_{m}(r)$ - радиус наибольшего шара, помешающегося в $\Omega^{r}[f]$. Очевидно, что условие С) будет выполнено, если функция $f(r)$ монотонно возрастает.

ТЕОРема 2. Пусть область $\Omega$ удовлетворяет условиям (11), (12), а области $\Omega_{i}, i=1,2,-$ условиям А), В), С). Пусть ${ }_{i}(t, x)$ - решение задачи (1)-(3)

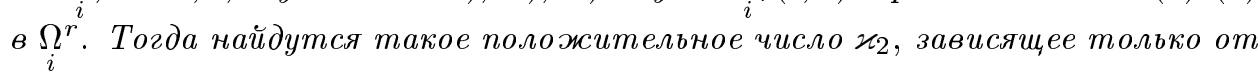
$n, \mu$ и $\nu$, и такие числа $T_{2}, M_{2}$, зависящие от области $\Omega$ и $R_{0}$, что для всех $t \geqslant T_{2}$ справедливо неравенство

$$
\int_{\substack{\Omega_{i}^{r}(t) \\ i}} u_{r_{i}(t)}^{2}(t, x) d x \geqslant M_{2} \exp \left(-\varkappa_{2}\left[\frac{r_{i}^{4}(t)}{t}\right]^{1 / 3}\right) .
$$


Лемма 1. Если области $\underset{i}{\Omega}, i=1,2$, удовлетворяют условиям В) и С), то

$$
\left[\frac{r_{i}^{4}(t)}{t}\right]^{1 / 3} \geqslant c_{0} \ln ^{2} t \quad \text { npu } \quad t \geqslant T^{*}, \quad i=1,2,
$$

где $c_{0}$ - положстельное число, $T^{*}$ - достаточно большое число.

ЗАмЕчанИЕ. Из (19) и (20) следует, что

$$
\max _{i} \int_{\substack{\Omega_{i}{ }^{i}(t) \\ i}} u_{r_{i}(t)}^{2}(t, x) d x \geqslant M_{2} \exp \left(-\varkappa_{2} \min _{i}\left[\frac{r_{i}^{4}(t)}{t}\right]^{1 / 3}\right)\|\varphi\|_{L_{2}(\Omega)}^{2}
$$

тем самым подтверждается точность оценки (17) в случае областей, удовлетворяющих условиям теоремы 2 , поскольку множитель $(1+t)$ пренебрежим по сравнению с $\exp \left(-\varkappa \min _{i}\left[\frac{r_{i}^{4}(t)}{t}\right]^{1 / 3}\right)$.

Отметим, что в отличие от задачи с первым краевым условием (7) переход от уравнения (1) к уравнению более высокого порядка добавляет новые технические трудности. В частности, для уравнения шестого порядка нам не удалось получить оценку сверху в случае уравнения с переменньми коэффициентами. Для уравнения вьшше шестого порядка мы не смогли получить оценку сверху даже для уравнения с постоянньми коэффициентами. Мы ограничимся рассмотрением уравнения вида

$$
u_{t}=\Delta^{3} u
$$

в цилиндрической области $D$ при следуюших краевых условиях:

$$
\left.u(t, x)\right|_{x \in \partial \Omega}=\left.\Delta u(t, x)\right|_{x \in \partial \Omega}=\left.\Delta^{2} u(t, x)\right|_{x \in \partial \Omega}=0
$$

и начальном условии (3).

Для простоты задачу $(21),(22),(3)$ будем рассматривать в области вида $\Omega=$ $\Omega[f]$, где $f$-монотонно возрастающая на $[0, \infty]$ функция такая, что граница области $\Omega$ принадлежит равномерно классу $C^{3}[0, \infty)$.

При исследовании поведения нормы решения смешанной задачи (21), (22), (3) использованы следующие обозначения:

$$
\Omega_{a}^{b}=\left\{x \in \Omega: a<x_{1}<b\right\}, \quad D_{a}^{b}=\left\{(t, x) \in D: a<x_{1}<b\right\} .
$$

Определим функцию $\rho_{m}(r), r>0$, как радиус наибольшего шара, лежащего в множестве $\Omega_{0}^{r}$. На область $\Omega$ мы налагаем следующие условия:

$$
\begin{aligned}
& \lim _{r \rightarrow \infty} \frac{r}{\rho_{m}(r)}=\infty, \\
& \lim _{r \rightarrow \infty} \rho_{m}(r)=\infty .
\end{aligned}
$$


Потребуем также, чтобы средняя кривизна $K$ границы $\partial \Omega$ удовлетворяла неравенству

$$
K(x) \leqslant 0, \quad x_{1} \geqslant R_{0} .
$$

Для области $\Omega$ вида (9) это условие записывается в виде

$$
f^{\prime \prime} \leqslant(n-2) \frac{1+\left(f^{\prime}\right)^{2}}{f} .
$$

В дальнейшем будем предполагать, что $r(t), t>0,-$ обратная к монотонно возрастающей непрерьвной функции $F(r)=r \rho_{m}^{5}(r), r>0$. Отметим, что $t=$ $r(t)\left(\rho_{m}(r(t))\right)^{5}$ и, следовательно,

$$
\left[\frac{r^{6}(t)}{t}\right]^{1 / 5}=\frac{t}{\rho_{m}^{6}(r(t))}=\frac{r(t)}{\rho_{m}(r(t))} .
$$

В следуюшей теореме $u(t, x)$ - решение задачи $(21),(22),(3)$ в области $\Omega$, а $u_{r}(t, x)$ - решение той же задачи в области $\Omega^{r}$ соответственно.

ТеОрема 3. Пусть область $\Omega=\Omega[f]$ с монотонной функиией $f$ удовлетворяет условиям (23), (24) и (25). Тогда найдутся такое полоэительное

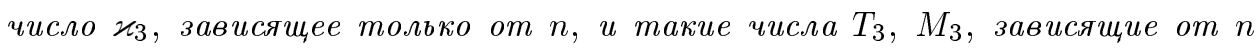
и $R_{0}\left(\operatorname{supp} \varphi \subset K_{R_{0}}\right)$, что для всех $t>T_{3}$ справедливо неравенство

$$
\int_{\Omega} u^{2}(t, x) d x \leqslant M_{3} \exp \left(-\varkappa_{3}\left[\frac{r^{6}(t)}{t}\right]^{1 / 5}\right)(1+t)\|\varphi\|_{L_{2}(\Omega)}^{2} .
$$

Такая же оценка справедлива и для функций $u_{r}(t, x)$ :

$$
\int_{\Omega^{r}} u_{r}^{2}(t, x) d x \leqslant M_{3} \exp \left(-\varkappa_{3}\left[\frac{r^{6}(t)}{t}\right]^{1 / 5}\right)(1+t)\|\varphi\|_{L_{2}(\Omega)}^{2} \quad \forall r \geqslant 2 R_{0} .
$$

Отметим, что из (26) и (23) следует соотношение

$$
\lim _{t \rightarrow \infty} \frac{r^{6}(t)}{t}=\infty
$$

т.е. экспонента в $(27)$ стремится к нулю при $t \rightarrow \infty$. В частности, для областей вида $\Omega\left[x_{1}^{\alpha}\right]$ оценка (27) примет вид

$$
\int_{\Omega} u^{2}(t, x) d x \leqslant M_{1} \exp \left(-\varkappa_{1} t^{(1-\alpha) /(1+5 \alpha)}\right), \quad t>T_{5}^{*} .
$$

ТЕорема 4. Пусть область $\Omega=\Omega[f]$ с монотонной функиией $f$ удовлетворяет условиям (23), (24), (25) и условиям А), В). Пусть $u_{r}(t, x)$ - решение задачи (21), (22), (3) в $\Omega^{r}$. Тогда найдутся такое положительное число ћ4, зависящее от $n$, и такие числа $T_{4}, M_{4}$, зависящие от $n$ и $R_{0}$, что для всех $t \geqslant T_{4}$ справедливо неравенство

$$
\int_{\Omega^{r(t)}} u_{r(t)}^{2}(t, x) d x \geqslant M_{4} \exp \left(-\varkappa_{4}\left[\frac{r^{6}(t)}{t}\right]^{1 / 5}\right) .
$$

Следуюшее утверждение аналогично лемме 1. 
ЛЕмма 2. Если область $\Omega$ удовлетворяет условию В), то

$$
\left[\frac{r^{6}(t)}{t}\right]^{1 / 5} \geqslant c_{0} \ln ^{2} t \quad \text { npu } \quad t \geqslant T^{*}
$$

ЗАМЕЧАнИЕ. Из (30) и (31) следует точность оценки (28) в случае областей, удовлетворяющих условиям теоремы 4 , поскольку множитель $(1+t)$ пренебрежим по сравнению с $\exp \left(-\varkappa\left[\frac{r^{6}(t)}{t}\right]^{1 / 5}\right)$.

1. Вспомогательные предложения для решений задач (1)-(3) и (21), (22), (3). Рассмотрим ряд функциональных пространств, необходимых для исследования задачи (1)-(3).

Пусть $W_{2,0}^{2}(\Omega)$ - подпространство $W_{2}^{2}(\Omega)$, плотным множеством в котором является $C_{0}^{2}(\bar{\Omega})$ - множество всех дважды непрерывно дифференцируемых в $\bar{\Omega}$ функций с ограниченным носителем, равных нулю на $\partial \Omega$. Через $W_{2,0}^{0,2}\left(D^{T}\right)$ будем обозначать пополнение множества функций $u \in C^{2}\left(\overline{D^{T}}\right)$, равных нулю на боковой поверхности цилиндра $[0, T] \times \partial \Omega$ и имеющих ограниченньй носитель, по норме

$$
\|u\|_{0,2\left(D^{T}\right)}=\left[\int_{D^{T}}\left(\left|\nabla^{2} u\right|^{2}+|\nabla u|^{2}+u^{2}\right) d x d t\right]^{1 / 2},
$$

в которую не включены производные от $u$ по $t ; W_{2,0}^{1,2}\left(D^{T}\right)$ - пополнение того же множества функций по норме

$$
\|u\|_{1,2\left(D^{T}\right)}=\left[\int_{D^{T}}\left(\left|\nabla^{2} u\right|^{2}+|\nabla u|^{2}+\left|u_{t}\right|^{2}+u^{2}\right) d x d t\right]^{1 / 2} .
$$

Обобщенным решением задачи (1)-(3) в $D^{T}$ будем называть функцию $u(t, x) \in$ $W_{2,0}^{0,2}\left(D^{T}\right)$, удовлетворяюшую тождеству:

$$
-\int_{D^{T}} u v_{t} d x d t+\int_{D^{T}} L u L v d x d t=\int_{\Omega} \varphi(x) v(0, x) d x
$$

для любой пробной функции $v \in W_{2,0}^{1,2}\left(D^{T}\right)$ такой, что $v(T, x)=0$.

Обобщенным решением задачи $(1)-(3)$ в $D$ будем называть функцию $u(t, x)$, совпадающую в общей области определения с решением задачи в $D^{T}$ при всех $T>0$.

Существование и единственность обобщенного решения задачи (1)-(3), а также задачи (21), (22), (3) доказываются обычными методами (см., например, [11], [12]).

Следуюшее утверждение аналогично предложению 1 из работы [13]. Доказательство, проведенное там для уравнения второго порядка, с соответствующими изменениями переносится на случай задачи (1)-(3). 
ПРЕДЛОЖЕНИЕ 1. Обобщенное решение $и(t, x)$ задачи (1)-(3) в D принадлежит классу $C\left([0, \infty) \rightarrow L_{2}(\Omega)\right)$ и для всех $t>0$ удовлетворяет тождеству

$$
\int_{\Omega} u^{2}(t, x) d x=\int_{\Omega} \varphi^{2} d x-2 \int_{0}^{t} \int_{\Omega}|L u|^{2} d x d t
$$

При исследовании решения задачи (21), (22), (3) будем использовать следующие функциональные пространства.

Пусть $C_{\Delta}^{3}(\Omega)$ - множество всех трижды непрерьвно дифференцируемых в $\bar{\Omega}$ функций $u(x)$ с ограниченным носителем таких, что $\left.u\right|_{\partial \Omega}=\left.\Delta u\right|_{\partial \Omega}=0$. Обозначим через $H_{\Delta}^{3}(\Omega)$ замыкание множества $C_{\Delta}^{3}(\Omega)$ в $W_{2}^{3}(\Omega)$. Через $H_{\Delta}^{0,3}\left(D^{T}\right)$ будем обозначать пополнение множества функций $u \in C^{3}\left(\overline{D^{T}}\right)$ таких, что $u(t) \in C_{\Delta}^{3}(\Omega)$ $\forall t \geqslant 0$ и имеюших ограниченный носитель, по норме

$$
\|u(t, x)\|_{\Delta\left(D^{T}\right)}^{(0,3)}=\left[\int_{D^{T}}\left(\left|\nabla^{3} u\right|^{2}+\left|\nabla^{2} u\right|^{2}+|\nabla u|^{2}+u^{2}\right) d x d t\right]^{1 / 2}
$$

$H_{\Delta}^{1,3}\left(D^{T}\right)$ - пополнение того же множества функций по норме

$$
\|u(t, x)\|_{\Delta\left(D^{T}\right)}^{(1,3)}=\left[\int_{D^{T}}\left(\left|\nabla^{3} u\right|^{2}+\left|\nabla^{2} u\right|^{2}+|\nabla u|^{2}+\left|u_{t}\right|^{2}+u^{2}\right) d x d t\right]^{1 / 2} .
$$

Обобщенным решением задачи $(21),(22),(3)$ в $D^{T}$ будем назьвать функцию $u(t, x) \in H_{\Delta}^{0,3}\left(D^{T}\right)$, удовлетворяющую тождеству:

$$
-\int_{D^{T}} u v_{t} d x d t+\int_{D^{T}}(\nabla \Delta u) \cdot(\nabla \Delta v) d x d t=\int_{\Omega} \varphi(x) v(0, x) d x
$$

для любой пробной функции $v \in H_{\Delta}^{1,3}\left(D^{T}\right)$ такой, что $v(T, x)=0$.

Обобшенным решением задачи (21), (22), (3) в $D$ будем называть функцию $u(t, x)$, совпадаюшую в обшей области определения с решением задачи в $D^{T}$ при всех $T>0$.

Следуюшее утверждение аналогично предложению 1.

ПрЕДЛОЖЕНИЕ 2. Обобщенное решение и(t, $x)$ задачи (21), (22), (3) в D принадлежит классу $C\left([0, \infty) \rightarrow L_{2}(\Omega)\right)$ и для всех $t>0$ удовлетворяет тождеству

$$
\int_{\Omega} u^{2}(t, x) d x=\int_{\Omega} \varphi^{2} d x-2 \int_{0}^{t} \int_{\Omega}|\nabla \Delta u|^{2} d x d t .
$$

Из предложений 1,2 следует, что функция $E(t)=\int_{\Omega} u^{2}(t, x) d x$ абсолютно непрерывна и монотонно не возрастает.

Установим ряд необходимых нам неравенств. Пусть $u \in H_{\Delta}^{3}(\Omega)$. Тогда, интегрируя по частям, мы можем получить

$$
\int_{\Omega}|\nabla u|^{2} d x=-\int_{\Omega}|u \Delta u| d x .
$$


Отсюда, применяя неравенство Коши, имеем

$$
\int_{\Omega}|\nabla u|^{2} d x \leqslant \frac{1}{2}\left[\varepsilon \int_{\Omega} u^{2} d x+\frac{1}{\varepsilon} \int_{\Omega}|\Delta u|^{2} d x\right]
$$

где $\varepsilon>0$ - произвольно. Используя аналогичные рассуждения, найдем

$$
\begin{aligned}
\int_{\Omega}|\Delta u|^{2} d x & =\int_{\Omega}|(\Delta u)(\operatorname{div} \nabla u)| d x=\int_{\Omega}|-(\nabla \Delta u) \cdot(\nabla u)| d x \\
& \leqslant \frac{1}{2}\left(\varepsilon_{1} \int_{\Omega}|\nabla u|^{2} d x+\frac{1}{\varepsilon_{1}} \int_{\Omega}|\nabla \Delta u|^{2} d x\right)
\end{aligned}
$$

где $\varepsilon_{1}>0$ - произвольно. Применив (35) к последнему неравенству, получим

$$
\int_{\Omega}|\Delta u|^{2} d x \leqslant \frac{\varepsilon_{1}}{2}\left(\frac{\varepsilon}{2} \int_{\Omega} u^{2} d x+\frac{1}{2 \varepsilon} \int_{\Omega}|\Delta u|^{2} d x\right)+\frac{1}{2 \varepsilon_{1}} \int_{\Omega}|\nabla \Delta u|^{2} d x .
$$

Полагая в последнем неравенстве $\varepsilon_{1}=2 \varepsilon$ и приводя подобные слагаемые, находим

$$
\int_{\Omega}|\Delta u|^{2} d x \leqslant \varepsilon^{2} \int_{\Omega} u^{2} d x+\frac{1}{2 \varepsilon} \int_{\Omega}|\nabla \Delta u|^{2} d x .
$$

Снова соединяя (35) и (36), видим, что

$$
\int_{\Omega}|\nabla u|^{2} d x \leqslant \varepsilon \int_{\Omega} u^{2} d x+\frac{1}{4 \varepsilon^{2}} \int_{\Omega}|\nabla \Delta u|^{2} d x .
$$

2. Доказательство теоремы 1 основано на следующем утверждении.

ПРЕДЛОЖЕНИЕ 3. Пусть область $\Omega$ удовлетворяет условиям (11), (12), коэффициенты уравнения (1) удовлетворяют условиям (5), (6) и выполнено условие (14). Пусть и $(t, x)$ - решение задачи (1)-(3). Тогда найдутся такие полохительнье числа $\delta_{i}, i=1,2$, зависящие только от $n, \mu$ и $\nu$, и такие числа $A, C_{i}, i=1,2$, зависящие от $n, \nu, \mu, R_{1}$ u $R_{0}\left(\operatorname{supp} \varphi \subset K\left(R_{0}\right)\right)$, что для всех $t>0$ и $r^{4}>$ At справедливо неравенство

$$
\int_{\substack{\Omega_{r}^{\infty} \\ n^{\infty}}} u^{2}(t, x) d x \leqslant C_{i} \exp \left(-\delta_{i}\left[\frac{r^{4}}{t}\right]^{1 / 3}\right)(1+t)\|\varphi\|_{L_{2}(\Omega)}^{2} .
$$

Аналогично, для решения и $u_{R}$ задачи (1)-(3) в области $\underset{i}{\Omega^{R}}$ справедлива оченка вида (38)

$$
\int_{\substack{\Omega_{i}^{R} \\ i_{R}}} u_{R}^{2}(t, x) d x \leqslant C_{i} \exp \left(-\delta_{i}\left[\frac{r^{4}}{t}\right]^{1 / 3}\right)(1+t)\|\varphi\|_{L_{2}(\Omega)}^{2} \quad \forall R>2 R_{0} .
$$


ДоКАЗАТЕЛЬСТво. Отметим, что при $r>R$ множество ${\underset{i}{r}}_{r}^{R}$ пусто и оценка (39) становится тривиальной. Так как доказательства оценок (38) и (39) проводятся аналогично, то мы изложим здесь только доказательство оценки (38).

Для функции $p(t, x) \in L_{2}\left(D^{T}\right)$ обозначим через $p_{h}$ осреднение Стеклова

$$
p_{h}=\frac{1}{h} \int_{t}^{t+h} p(\tau, x) d \tau, \quad t+h<T
$$

Пусть $v \in C^{2}\left(\overline{D^{T}}\right), v(t) \in C_{0}^{2}(\bar{\Omega}) \forall t \geqslant 0,-$ произвольная пробная функция с ограниченным носителем такая, что $v=0$ при $t \geqslant T-h$. Продолжим ее гладким образом на значения $t \leqslant 0$. Обозначим через $\widetilde{v}$ продолжение функции $v$. Подставив в тождество $(32)$ в качестве пробной функции $\widetilde{v}_{-h}, h>0$, получим

$$
\int_{D^{T}}\left(-u\left(\widetilde{v}_{-h}\right)_{t}+L u L\left(\widetilde{v}_{-h}\right)\right) d x d t=\int_{\Omega} \varphi \widetilde{v}_{-h}(0, x) d x .
$$

Произведя вычисления на основе свойств осреднения Стеклова

$$
\int_{-\infty}^{+\infty}\left(u, v_{-h}\right)_{L_{2}(\Omega)} d t=\int_{-\infty}^{+\infty}\left(u_{h}, v\right)_{L_{2}(\Omega)} d t, \quad(L u)_{h}=L u_{h}
$$

нетрудно получить соотношение

$$
\begin{aligned}
& \int_{D^{T-h}}\left(v\left(u_{h}\right)_{t}+L v L u_{h}\right) d x d t \\
& \quad=\int_{\Omega} \varphi \widetilde{v}_{-h}(0, x) d x-\int_{-h}^{0} \int_{\Omega}\left(u_{h}\right)_{t} \widetilde{v}(t) d x d t-\int_{-h}^{0} \int_{\Omega} L u_{h} L \widetilde{v}(t) d x d t
\end{aligned}
$$

в котором функция $u(t, x)$ предполагается продолженной нулем на отрицательные значения $t$. Докажем, что правая часть (40) равна нулю при любом продолжении функции $v(t, x)$. Выберем такую последовательность $\widetilde{v}_{k}$ продолжений функции $v(t, x)$, чтобы последовательность $\int_{-h}^{0} \int_{\Omega}\left[\widetilde{v}_{k}^{2}+\left|L \widetilde{v}_{k}\right|^{2}\right] d x d t$ стремилась к нулю при $k \rightarrow \infty$. Подставляя в равенство (40) функции $\widetilde{v}_{k}$, переходя к пределу при $k \rightarrow \infty$, получим

$$
\int_{D^{T-h}}\left(v\left(u_{h}\right)_{t}+L v L u_{h}\right) d x d t=0 .
$$

Отметим, что равенство (41) справедливо и для любой функции $v \in W_{2,0}^{0,2}\left(D^{T}\right)$, $v(t, x)=0$ при $t \geqslant T-h$.

Пусть $s(r)$ - гладкая неотрицательная функция, равная нулю при $r \leqslant 0$ и единице при $r \geqslant 1$. Определим срезающую функцию $\eta(x, r, \rho)$ равенством

$$
\eta(x, r, \rho)=s\left(\frac{x_{1}-r}{\rho}\right)
$$


Пусть $t_{1}<T$ и $h \in\left(0, T-t_{1}\right)$. Подставив в (41) пробную функцию

$$
v(t, x)= \begin{cases}\eta^{2} u_{h}, & t<t_{1}, \\ 0, & t \geqslant t_{1},\end{cases}
$$

получим

$$
\int_{D^{t_{1}}}\left[\frac{1}{2}\left(\left(u_{h}^{2} \eta^{2}\right)_{t}+L\left(\eta^{2} u_{h}\right) L u_{h}\right)\right] d x d t=0 .
$$

Согласно предложению 1 решение задачи (1)-(3) принадлежит классу $C([0, \infty) \rightarrow$ $\left.L_{2}(\Omega)\right)$, следовательно,

$$
\int_{\Omega} u_{h}^{2}\left(t_{1}, x\right) \eta^{2} d x \rightarrow \int_{\Omega} u^{2}\left(t_{1}, x\right) \eta^{2} d x, \quad h \rightarrow 0
$$

Учитывая, что

$$
\int_{D^{t_{1}}}\left(\eta^{2} u_{h}^{2}\right)_{t} d x d t=\int_{\Omega}\left[u_{h}^{2}\left(t_{1}, x\right)-u_{h}^{2}(0, x)\right] \eta^{2} d x
$$

после предельного перехода в равенстве $(42)$ при $h \rightarrow 0$ получим

$$
\frac{1}{2} \int_{\Omega}\left[u^{2}\left(t_{1}, x\right)-u^{2}(0, x)\right] \eta^{2} d x+\int_{D^{t_{1}}} L u L\left(\eta^{2} u\right) d x d t=0
$$

Прибавим к обеим частям этого равенства интеграл $\int_{D^{t_{1}}}(L(\eta u))^{2} d x d t$. Тогда из (43) будем иметь

$\frac{1}{2} \int_{\Omega}\left[u^{2}\left(t_{1}, x\right)-\varphi^{2}\right] \eta^{2} d x+\int_{D^{t_{1}}}(L(\eta u))^{2} d x d t=\int_{D^{t_{1}}}\left[(L(\eta u))^{2}-L u L\left(\eta^{2} u\right)\right] d x d t$.

Рассмотрим разность - подынтегральное выражение, стоящее в правой части равенства (44):

$$
\begin{aligned}
(L(\eta u))^{2} & -L u L\left(\eta^{2} u\right)=\left(\eta L u+u L \eta+2 \sum_{i, j=1}^{n} a_{i j} \eta_{x_{i}} u_{x_{j}}\right)^{2} \\
& -L u\left(\eta^{2} L u+u L \eta^{2}+2 \sum_{i, j=1}^{n} a_{i j} \eta_{x_{i}}^{2} u_{x_{j}}\right) \\
= & (u L \eta)^{2}+\left(2 \sum_{i, j=1}^{n} a_{i j} \eta_{x_{i}} u_{x_{j}}\right)^{2}+4 u \sum_{i, j=1}^{n} a_{i j} \eta_{x_{i}} u_{x_{j}} L \eta \\
& -2 u \sum_{i, j=1}^{n} a_{i j} \eta_{x_{i}} \eta_{x_{j}} L u .
\end{aligned}
$$


Учитьвая вид функции $\eta$, имеем $\nabla \eta=0$ вне области $\underset{i}{\Omega_{r}^{r+\rho}}$. Тогда, принимая во внимание условие (6), получим

$$
|\nabla \eta| \leqslant \frac{c_{0}}{\rho}, \quad|L \eta|=\left|\sum_{i, j=1}^{n} a_{i j} \eta_{x_{i} x_{j}}+\sum_{i, j=1}^{n}\left(a_{i j}\right)_{x_{j}} \eta_{x_{i}}\right| \leqslant c_{1}\left(\frac{1}{\rho^{2}}+\frac{g(r)}{\rho}\right) .
$$

Взяв $\rho<1 / g(r)$, будем иметь $|L \eta| \leqslant 2 c_{1} / \rho^{2}$. Здесь и далее $c_{i}$ и $c_{i}^{\prime}$ зависят от $n, \mu$ и $\nu$.

Теперь из равенства (44), благодаря включению $\operatorname{supp} \varphi \subset K\left(R_{0}\right)$, для всех $r>R_{0}, 0<\rho<1 / g(r)$ можем получить

$$
\begin{aligned}
& \frac{1}{2} \int_{\substack{\Omega_{r}^{\infty} \\
n_{r}}} u^{2}\left(t_{1}, x\right) \eta^{2} d x+\int_{0}^{t_{1}} \int_{\substack{\Omega_{i}^{\infty} \\
r_{r}}}(L(\eta u))^{2} d x d t \\
& \leqslant c_{2} \int_{0}^{t_{1}} \int_{\substack{\Omega_{r}^{r+\rho} \\
\rho^{r}}}\left[\frac{u^{2}}{\rho^{4}}+\frac{|\nabla u|^{2}}{\rho^{2}}+\frac{1}{\rho^{2}}\left(\varepsilon^{2} u^{2}+\frac{|L u|^{2}}{\varepsilon^{2}}\right)\right] d x d t .
\end{aligned}
$$

Покажем, например, как оценивается слагаемое

$$
I_{4}=\int_{D^{t_{1}}} 2 u \sum_{i, j=1}^{n} a_{i j} \eta_{x_{i}} \eta_{x_{j}} L u d x d t
$$

Учитывая вид функции $\eta$ и оценки для функций $a_{i j}, \eta$, устанавливаем, что

$$
I_{4} \leqslant \int_{0}^{t_{1}} \int_{\Omega_{i}^{r+\rho}} \frac{c_{2}^{\prime}}{\rho^{2}}|u L u| d x d t
$$

Далее, применяя неравенство Коши, имеем

$$
I_{4} \leqslant c_{2}^{\prime} \int_{0}^{t_{1}} \int_{\substack{\Omega_{r}^{r+\rho} \\ r+\rho}} \frac{1}{\rho^{2}}\left(\varepsilon^{2} u^{2}+\frac{|L u|^{2}}{\varepsilon^{2}}\right) d x d t .
$$

Используя неравенство (5), интегрируя по частям и принимая во внимание (2), установим неравенство

$$
\begin{aligned}
\int_{\Omega}|\nabla u|^{2} d x & \leqslant \nu^{-1} \int_{\Omega} \sum_{i, j=1}^{n} a_{i j} u_{x_{i}} u_{x_{j}} d x=-\nu^{-1} \int_{\Omega} u L u d x \\
& \leqslant \frac{1}{2 \nu}\left(\varepsilon \int_{\Omega} u^{2} d x+\frac{1}{\varepsilon} \int_{\Omega}(L u)^{2} d x\right) .
\end{aligned}
$$

Здесь $\varepsilon>0$ - произвольно, неравенство верно для всех $t>0$.

Положим для краткости записи $\eta_{r}=\eta\left(x_{1}, r, \rho\right), \eta_{r-\rho}=\eta\left(x_{1}, r-\rho, \rho\right)$. В силу выбора функции $\eta$ к функции $\eta_{r-\rho} u$ применимо неравенство (46) в области $\underset{i}{\Omega_{r-\rho}^{\infty}}$ :

$$
\int_{\substack{\Omega_{r}^{\infty} \\ i}}|\nabla u|^{2} d x \leqslant \int_{\substack{\Omega_{r-\rho}^{\infty} \\ i}}\left|\nabla\left(\eta_{r-\rho} u\right)\right|^{2} d x \leqslant \frac{1}{2 \nu} \int_{\substack{\Omega_{i}^{\infty}-\rho \\ i}}\left(\varepsilon^{2} \eta_{r-\rho}^{2} u^{2}+\frac{1}{\varepsilon^{2}}\left|L\left(\eta_{r-\rho} u\right)\right|^{2}\right) d x .
$$


Теперь (45) можно привести к виду

$$
\begin{aligned}
& \frac{1}{2} \int_{\substack{\Omega_{r}^{\infty} \\
n^{2}}} u^{2}\left(t_{1}, x\right) \eta_{r}^{2} d x+\int_{0}^{t_{1}} \int_{\Omega_{i}^{\infty}}\left(L\left(\eta_{r} u\right)\right)^{2} d x d t \\
& \leqslant c_{3} \int_{0}^{t_{1}} \int_{\substack{\Omega_{i} \infty \\
r}}\left[\eta_{r-\rho}^{2} u^{2}\left(\frac{1}{\rho^{4}}+\frac{\varepsilon^{2}}{\rho^{2}}\right)+\frac{1}{\varepsilon^{2} \rho^{2}}\left|L\left(\eta_{r-\rho} u\right)\right|^{2}\right] d x .
\end{aligned}
$$

Будем рассматривать такие $\varepsilon$ и $\rho$, что $\varepsilon \rho \geqslant 1$, тогда

$$
\frac{1}{\rho^{4}}=\frac{\varepsilon^{4}}{\varepsilon^{4} \rho^{4}} \leqslant \frac{\varepsilon^{4}}{\varepsilon \rho}, \quad \frac{\varepsilon^{2}}{\rho^{2}} \leqslant \frac{\varepsilon^{4}}{\varepsilon \rho}, \quad \frac{1}{\varepsilon^{2} \rho^{2}} \leqslant \frac{1}{\varepsilon \rho} .
$$

В итоге (47) примет вид

$$
\begin{aligned}
& \frac{1}{2} \int_{\substack{\Omega_{r}^{\infty}\\
}} u^{2}\left(t_{1}, x\right) \eta_{r}^{2} d x+\int_{0}^{t_{1}} \int_{\Omega_{i}^{\infty}}\left(L\left(\eta_{r} u\right)\right)^{2} d x d t \\
& \quad \leqslant \frac{2 c_{3}}{\varepsilon \rho} \int_{0}^{t_{1}} \int_{\substack{\Omega_{r}^{\infty}-\rho\\
}}\left[\varepsilon^{4} \eta_{r-\rho}^{2} u^{2}+\left|L\left(\eta_{r-\rho} u\right)\right|^{2}\right] d x .
\end{aligned}
$$

Введем обозначение

$$
H_{r}(t, \rho)=\int_{\Omega_{i}^{\infty}} u^{2}(t, x) \eta_{r}^{2} d x+\int_{0}^{t} \int_{\Omega_{i}^{\infty}}\left(L\left(\eta_{r} u\right)\right)^{2} d x d t .
$$

Тогда из (48) последует

$$
H_{r}(t, \rho) \leqslant \frac{c_{4}}{\varepsilon \rho}\left(\varepsilon^{4} \int_{0}^{t} H_{r-\rho}(\tau, \rho) d \tau+H_{r-\rho}(t, \rho)\right) .
$$

Докажем неравенство

$$
H_{r}(t, \rho) \leqslant A_{0}(1+t)
$$

для $t \geqslant 0, r \geqslant R_{0}, 1 \leqslant \rho \leqslant 1 / g(r)$, где $A_{0}=c_{4}^{\prime}\|\varphi\|^{2}$ - константа. Используя неравенство (45) при $\rho \geqslant 1, \varepsilon=1$, имеем

$$
H_{r}(t, \rho) \leqslant c_{5} \int_{0}^{t} \int_{\Omega}\left[u^{2}+|\nabla u|^{2}+(L u)^{2}\right] d x d t
$$

Применив к последнему выражению (46), получаем

$$
H_{r}(t, \rho) \leqslant c_{5}^{\prime} \int_{0}^{t} \int_{\Omega}\left[u^{2}+(L u)^{2}\right] d x d t
$$

Теперь, используя (33), легко получим требуемое неравенство (50). 
Далее, индукцией по $k$ установим неравенство

$$
H_{r+k \rho}(t, \rho) \leqslant\left(\frac{2 c_{4}}{\rho}\right)^{k} t^{k / 4}\left(\frac{3 ! 4^{k}}{(3+k) !}\right)^{1 / 4} A_{0}(1+t)
$$

для $t \geqslant 0, r \geqslant R_{0}, 1 \leqslant \rho \leqslant 1 / g(r)$. Пусть

$$
\varepsilon=\left(\frac{4+k}{4 t}\right)^{1 / 4}
$$

Если окажется, что $\varepsilon \rho<1$, то в индуктивном предположении $(51)$ вместо $r$ можно взять $r+\rho$ :

$$
\begin{aligned}
H_{r+(k+1) \rho}(t, \rho) & \leqslant \frac{2 c_{4}}{\varepsilon \rho} H_{(r+\rho)+k \rho}(t, \rho) \\
& \leqslant\left(\frac{2 c_{4}}{\rho}\right)^{k+1} t^{(k+1) / 4}\left(\frac{3 ! 4^{k+1}}{(4+k) !}\right)^{1 / 4} A_{0}(1+t) .
\end{aligned}
$$

При $\varepsilon \rho \geqslant 1$ справедливо неравенство (49):

$$
\begin{aligned}
H_{r+(k+1) \rho}(t, \rho) & \leqslant \frac{c_{4}}{\varepsilon \rho}\left(\varepsilon^{4} \int_{0}^{t} H_{r+k \rho}(\tau, \rho) d \tau+H_{r+k \rho}(t, \rho)\right) \\
& \leqslant \frac{c_{4}}{\varepsilon \rho}\left(\frac{2 c_{4}}{\rho}\right)^{k}\left(\frac{3 ! 4^{k}}{(3+k) !}\right)^{1 / 4} A_{0}\left(\varepsilon^{4} \int_{0}^{t} \tau^{k / 4}(1+t) d \tau+t^{k / 4}(1+t)\right) \\
& =\left(\frac{2 c_{4}}{\rho}\right)^{k+1} t^{(k+1) / 4}\left(\frac{3 ! 4^{k+1}}{(4+k) !}\right)^{1 / 4} A_{0}(1+t) .
\end{aligned}
$$

Таким образом, (51) для $k+1$ установлено. Индукция завершена.

Используя неравенство Стирлинга $n !>\left(c_{6} / \sqrt{n}\right)(n / e)^{n}$, где $c_{6}<1 / \sqrt{2 \pi}$, из неравенства (51) несложно получить

$$
H_{r+k \rho}(t, \rho) \leqslant \exp \left(-\frac{k}{4} \ln \left(\frac{k \rho^{4}}{c_{7} t}\right)\right) A_{1}(1+t) .
$$

Положим $\rho=r /(2(k+1))$, где $k$ - наибольшее натуральное число такое, что $r^{4}>16 c_{7}(k+1)^{3} e t, r \geqslant 2 R_{0}$. Существование такого натурального $k$ обеспечивается неравенством $r^{4}>A t$ и выбором достаточно большого $A$. Выясним, для каких $r$ и $t$ выполнены неравенства $1 \leqslant \rho \leqslant 1 / g(r)$. Имеем, с одной стороны,

$$
(k+1)>\left(\frac{r^{4}}{16 c_{7} e t}\right)^{1 / 3}-1>\frac{1}{2}\left(\frac{r^{4}}{16 c_{7} e t}\right)^{1 / 3} .
$$

Отсюда легко видно, что $\rho<\left(16 c_{7} e t / r\right)^{1 / 3}$. С другой стороны,

$$
\rho>\frac{1}{2}\left(\frac{16 c_{7} e t}{r}\right)^{1 / 3}>1
$$


Поэтому для значений времени, удовлетворяющих неравенствам

$$
8 \leqslant \frac{16 c_{7} e t}{r} \leqslant \frac{1}{g^{3}(r)}
$$

справедливы ограничения, наложенные на $\rho$. В дальнейшем в (54) мы будем выбирать $t=r \lambda_{i}^{-3 / 2}(r), i=1,2$. Чтобы это было допустимым, необходимо при каждом $i$ соблюдение неравенства

$$
8 \leqslant \frac{16 c_{7} e r \lambda_{i}^{-3 / 2}(r)}{r} \leqslant \frac{1}{g^{3}(r)}
$$

при достаточно больших значениях $r$. Из правого неравенства мы получим ограничение на функцию $g(r)$ :

$$
g(r) \leqslant V \min _{i}\left\{\lambda_{i}^{1 / 2}(r)\right\}
$$

где константа $V$ зависит только от $n, \nu$ и $R_{0}$.

По выбору $k$ имеем $\rho^{4}>c_{7} e t /(k+1)$, поэтому $k \rho^{4} /\left(c_{7} t\right)>e / 2$. Ввиду (53) из неравенства (52) выводим

$$
H_{r-\rho}(t, \rho)=H_{r / 2+k \rho}(t, \rho) \leqslant \exp \left(-\delta_{0}\left(\frac{r^{4}}{t}\right)^{1 / 3}(1-\ln 2)\right) A_{2}(1+t) .
$$

Отсюда легко следует неравенство

$$
H_{r-\rho}(t, \rho) \leqslant \exp \left(-\delta_{1}\left(\frac{r^{4}}{t}\right)^{1 / 3}\right) A_{2}(1+t) .
$$

Очевидно, что

$$
\int_{\substack{\Omega^{\infty} \\ r^{\infty}}} u^{2} d x \leqslant H_{r-\rho}(t, \rho) .
$$

При помощи (55) получим неравенство (38) предложения 3.

ДОКАЗАТЕЛЬСТво ТЕОРЕмЫ 1. Оценки (16) и (17) получаются единообразно с использованием предложения 3 , поэтому ограничимся доказательством оценки (16).

Наряду с уже рассмотренньпи функциями $\underset{i}{\lambda}(r), i=1,2$, определим функции $\mu_{i}(r), r>0, i=1,2$,

$$
\mu_{i}(r)=\inf _{\substack{\circ \\ v \in W_{2}^{1}(\Omega)}} \int_{\substack{\Omega_{0}^{r} \\ i}}|\nabla v|^{2} d x\left[\int_{\substack{\Omega_{0}^{r} \\ \gamma^{r}}} v^{2} d x\right]^{-1} .
$$

В работе [10] доказано, что сушествует число $c_{0}>0$ такое, что для любой области $\Omega_{i}$, удовлетворяющей условиям (11) и (12), найдется число $r_{0}>1$ такое, что при всех $r \geqslant r_{0}$ справедливо неравенство

$$
c_{0} \lambda(r) \leqslant \mu_{i}(r)
$$


Число $r_{0}>1$ выберем такое, чтобы при всех $r \geqslant r_{0}$ выполнялось также неравенство

$$
\min _{i}\left\{c_{0} \lambda(r)\right\} \leqslant \mu^{*} .
$$

Используя определение функции $\mu_{i}(r)$, числа $\mu^{*}$ и неравенство $(46)$, находим

$$
\begin{aligned}
& \min _{i}\left(c_{0} \lambda(r)\right)\left(\int_{\Omega} u^{2} d x-\sum_{i=1}^{2} \int_{\substack{\Omega_{r}^{\infty} \\
r}} u^{2} d x\right) \leqslant \mu^{*} \int_{\substack{\Omega \\
0}} u^{2} d x+\sum_{i=1}^{2} \mu_{i}(r) \int_{\substack{\Omega_{0}^{r} \\
i}} u^{2} d x \\
& \leqslant \int_{\substack{\Omega \\
0}}|\nabla u|^{2} d x+\sum_{i=1}^{2} \int_{\substack{\Omega_{0}^{r} \\
i^{r}}}|\nabla u|^{2} d x \leqslant 2 \int_{\Omega}|\nabla u|^{2} d x \\
& \leqslant \frac{1}{\nu}\left(\varepsilon \int_{\Omega} u^{2} d x+\frac{1}{\varepsilon} \int_{\Omega}(L u)^{2} d x\right) .
\end{aligned}
$$

Взяв $\varepsilon=\frac{\nu}{2} \min _{i}\left(c_{0} \underset{i}{\lambda}(r)\right)$, из последнего выражения получим

$$
\left(\frac{1}{2} \int_{\Omega} u^{2} d x-\sum_{i=1}^{2} \int_{\Omega_{i}^{\infty}} u^{2} d x\right) \leqslant 2\left[\nu \min _{i}\left(c_{0} \lambda(r)\right)\right]^{-2} \int_{\Omega}(L u)^{2} d x
$$

Обозначим

$$
\beta=\sum_{i=1}^{2} \max _{[0, T]} \int_{\Omega_{i}^{\infty}} u^{2} d x, \quad \lambda^{*}=\frac{1}{2}\left[\nu \min _{i}\left(c_{0} \lambda(r)\right)\right]^{2}
$$

Согласно предложению 1 функция $E(t)=\int_{\Omega} u^{2}(t, x) d x$ абсолютно непрерывна и

$$
\frac{d E}{d t}=-2 \int_{\Omega}(L u)^{2} d x
$$

Из установленного выше неравенства находим

$$
\lambda^{*}(E(t)-2 \beta) \leqslant-\frac{d E}{d t} .
$$

Отсюда легко получить, что

$$
E(t)-2 \beta \leqslant \exp \left(-\lambda^{*} t\right)(E(0)-2 \beta)
$$

Поскольку $E(0)=\int_{\Omega} \varphi^{2} d x$, из последнего неравенства будем иметь

$$
E(t) \leqslant 2 \beta+\exp \left(-\lambda^{*} t\right)\|\varphi\|^{2} .
$$


Согласно предложению 3 при всех $t>0, r \geqslant \max \left\{2 R_{0}, r_{0}, R_{1}, \sqrt[4]{A t}\right\}$ имеем

$$
\begin{aligned}
\beta & \leqslant \sum_{i=1}^{2} C_{i} \exp \left(-\delta_{i}\left[\frac{r^{4}}{t}\right]^{1 / 3}\right)(1+t)\|\varphi\|_{L_{2}(\Omega)}^{2} \\
& \leqslant M_{1} \exp \left(-\varkappa_{1} \min _{i}\left[\frac{r^{4}}{t}\right]^{1 / 3}\right)(1+t)\|\varphi\|_{L_{2}(\Omega)}^{2},
\end{aligned}
$$

где $M_{1}=2 \max _{i}\left\{C_{i}\right\}, \varkappa_{1}=\min _{i}\left\{\delta_{i}\right\}$. Теперь из неравенства (58) получим

$$
\begin{aligned}
\int_{\Omega} u^{2}(t, x) d x \leqslant & M_{1} \exp \left(-\varkappa_{1} \min _{i}\left[\frac{r^{4}}{t}\right]^{1 / 3}\right)(1+t)\|\varphi\|_{L_{2}(\Omega)}^{2} \\
& +\exp \left(-\left[\nu \min _{i}\left(c_{0}{\underset{i}{i}}_{(r)}\right)\right]^{2} t\right)(1+t)\|\varphi\|_{L_{2}(\Omega)}^{2} .
\end{aligned}
$$

Отметим, что в силу соотношения (18) сушествует такое число $T>0$, что при всех $t \geqslant T$ справедливо неравенство $r_{i}(t) \geqslant \max \left\{2 R_{0}, r_{0}, R_{1}, \sqrt[4]{A t}\right\}$. Подставляя в (59) $r=r_{i}(t)$ и используя равенства (15), получим оценку (16) теоремы 1.

\section{3. Оценка снизу для решения задачи (1)-(3).}

ДоКАЗАТЕЛЬСТВо ЛЕММЫ 1. В работе [14] показано, что функции $\underset{i}{\lambda}(r), i=1,2$, подчиняются неравенству

$$
c^{-1} \rho_{i}^{-2}(r) \leqslant \lambda_{i}(r) \leqslant c \rho_{i}^{-2}(r), \quad c>1, \quad i=1,2 .
$$

Ввиду условия С) имеем также неравенство

$$
\sigma^{-2} c^{-1} f_{i}^{-2}(r) \leqslant \underset{i}{\lambda}(r), \quad r \geqslant P
$$

Из условия В) легко получить, что

$$
\frac{1}{f_{i}(r)} \geqslant \frac{1}{C r} \int_{P}^{r} \frac{d \rho}{f_{i}(\rho)} \geqslant \frac{1}{C r} \int_{P}^{2 P} \frac{d \rho}{f_{i}(\rho)}=\frac{\gamma}{r}, \quad r \geqslant 2 P .
$$

Здесь и далее в доказательстве леммы постоянная $\gamma$ зависит только от функции $f_{i}$ и постоянных $C$ и $P$ из условия В), $\sigma$ из условия С) и $c$ из (60), поэтому для удобства чтения мы опустим индексы, подразумевая в каждом случае свою константу $\gamma$. Теперь, используя (62), будем иметь

$$
\int_{2 P}^{r} \frac{d \rho}{f_{i}(\rho)} \geqslant \gamma \int_{2 P}^{r} \frac{d \rho}{\rho}=\gamma \ln \left(\frac{r}{2 P}\right), \quad r \geqslant 2 P .
$$

Следовательно,

$$
\frac{1}{f_{i}(r)} \geqslant \frac{1}{C r} \int_{P}^{r} \frac{d \rho}{f_{i}(\rho)} \geqslant \frac{\gamma}{r} \ln \left(\frac{r}{2 P}\right), \quad r \geqslant 2 P .
$$


Отсюда

$$
\int_{2 P}^{r} \frac{d \rho}{f_{i}(\rho)} \geqslant \int_{2 P}^{r} \frac{\gamma}{s} \ln \left(\frac{s}{2 P}\right) d s=\gamma \ln ^{2}\left(\frac{r}{2 P}\right), \quad r \geqslant 2 P .
$$

По неравенству (61) и условию В) имеем

$$
r \underset{i}{\lambda_{i}^{1 / 2}}(r) \geqslant \frac{\gamma r}{f_{i}(r)} \geqslant \gamma \ln ^{2}\left(\frac{r}{2 P}\right), \quad r \geqslant 2 P .
$$

Положим $t=r \lambda_{i}^{-3 / 2}(r)$. Используя $(61)$ и $(62)$, получим

$$
t \leqslant \gamma r f_{i}^{3}(r) \leqslant \gamma r^{4}
$$

Отсюда $r \geqslant \gamma t^{1 / 4}$. Следовательно,

$$
r \lambda_{i}^{1 / 2}(r) \geqslant \gamma \ln ^{2}\left(\frac{\gamma t^{1 / 4}}{2 P}\right) \geqslant c_{0} \ln ^{2} t
$$

Теперь из равенств (15) следует справедливость утверждения леммы.

Доказательство леммы 2 вполне аналогично доказательству леммы 1 и поэтому опускается.

ДоКАЗАТЕЛЬСтво теоремы 2. Зафиксируем произвольное $i=1,2$. Пусть функция $v(x)$ - первая нормированная в $L_{2} \underset{i}{\left(\Omega^{r}\right)}$ собственная функция оператора $L$ :

$$
L v=-\underset{i}{\lambda_{L}}(r) v,\left.\quad v\right|_{\substack{\partial \Omega_{i}^{r}\\}}=0
$$

Если $\left.B \underset{i}{\rho_{m}}, \underset{i}{z}\right) \subset \underset{i}{\Omega^{r}}-$ максимальный шар в $\underset{i}{\Omega^{r}}$, то, как известно,

$$
\left.\lambda_{i} L\left(\underset{i}{\Omega^{r}}\right) \leqslant \underset{i}{\lambda_{L}}\left(B \underset{i}{\rho_{m}}, \underset{i}{z}\right)\right)
$$

Полагая $W_{i}=\left\{u \in \stackrel{\circ}{W} \underset{2}{1}\left(\Omega \backslash \underset{i}{\Omega_{r}^{\infty}}\right): \int_{\substack{\Omega_{0}^{r} \\ x^{r}}} u^{2} d x=1\right\}$, используя неравенство (5), оценим функцию $\lambda_{i}(r)$ :

$$
\lambda_{i} L(r)=\inf _{u \in W_{i}} \int_{\substack{\Omega_{0}^{r} \\ i}}\left|a_{i j}(x) u_{x_{i}} u_{x_{j}}\right| d x \leqslant \mu \inf _{u \in W_{i}} \int_{\substack{\Omega_{0}^{r} \\ i}}|\nabla u|^{2} d x=\mu \lambda_{i}(r), \quad i=1,2 .
$$

Выберем ось $O x_{1}$ направленной вдоль некоторого луча $s_{i}$ - оси вращения области $\Omega_{i}$. Для собственной функции $v$ в работе [15] установлено неравенство Гарнака в следующей форме. Для произвольного шара $B(\rho, z) \subset \underset{i}{\Omega_{0}^{r}}$ справедливо неравенство

$$
\max _{B(\rho / 2, z)} v \leqslant H \min _{B(\rho / 2, z)} v
$$

где число $H$ зависит только от $n, \nu, \mu$. 
Шар $B(\rho, z)$ будем называть допустимым для области $\underset{i}{\Omega^{r}}$, если

1) $z \in O x_{1}$

2) $B(2 \rho, z) \subset \Omega^{r}$,

3) $\forall \varepsilon>0 B(2 \rho+\varepsilon, z) \not \subset \underset{i}{\Omega^{r}}$.

Зафиксируем произвольный индекс $i$ и выберем произвольную точку $z_{0} \in \underset{i}{\Omega \text { на }}$

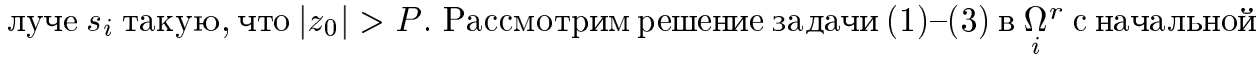
функцией $\chi_{B\left(\rho_{0}, z_{0}\right)}$, где $B\left(\rho_{0}, z_{0}\right)$ - допустимый шар. Оно представляется в виде

$$
u_{r}(t, x)=\sum_{j=1}^{\infty} c_{j} \exp \left(-\lambda_{j}(r) t\right) v_{j}(x)
$$

где $v_{j}(x)$ - собственные функции оператора $L^{2}$ в области $\underset{i}{\Omega^{r}}$. В нашем случае собственная функция $v_{1}$ оператора $L^{2}$ одновременно является первой собственной функцией оператора $L$, причем $\lambda_{1}(r)=\lambda_{i}^{2}(r)$. Используя (64), получим

$$
\int_{\substack{\Omega^{r} \\ \text { r }}} u_{r}^{2} d x \geqslant c_{1}^{2} \exp \left(-2 \lambda_{1}(r) t\right) \geqslant c_{1}^{2} \exp \left(-2 \mu^{2} \lambda_{i}^{2}(r) t\right)
$$

где

$$
c_{1}=\int_{\substack{\Omega^{r} \\ i}} \chi_{B\left(\rho_{0}, z_{0}\right)} v_{1} d x
$$

Чтобы оценить снизу число $c_{1}$, надо оценить минимум собственной функции $v_{1}(x)$ по шару $B\left(\rho_{0}, z_{0}\right)$. Построим цепочку допустимых шаров $B\left(\rho_{k}, z_{k}\right), k=0, \ldots, q$, $z_{k}$ - центры, $y_{k}-$ правые точки касания этих шаров. Тогда

$$
\min _{B\left(\rho_{0}, z_{0}\right)} v \geqslant H^{-1} v\left(y_{0}\right) .
$$

Кроме того, $v\left(y_{k-1}\right) \geqslant H^{-1} v\left(y_{k}\right)$, следовательно,

$$
\min _{B\left(\rho_{0}, z_{0}\right)} v \geqslant H^{-(q+1)} v\left(y_{q}\right) .
$$

Цепочка допустимых шаров будет построена таким образом, что значение $v\left(y_{q}\right)$ будет эффективно оценено снизу. Более точно, в качестве $y_{q}$ будет выбрана точка

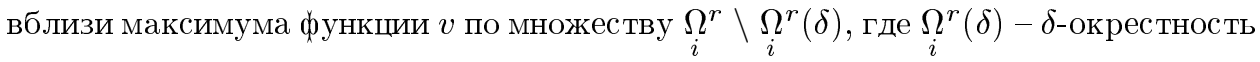
границы $\underset{i}{\partial \Omega^{r}}$ :

$$
\underset{i}{\Omega^{r}}(\delta)=\left\{x \in \underset{i}{\Omega^{r}}: \operatorname{dist}\left(x, \underset{i}{\partial \Omega^{r}}\right)<\delta\right\} .
$$

Оценим этот максимум снизу. По неравенству $Ф$ ридрихса-Стеклова имеем

$$
\begin{aligned}
\int_{\substack{\Omega^{r}(\delta) \\
v^{2}}} v^{2} d x & \leqslant \delta^{2} \int_{\substack{\Omega_{i}^{r}(\delta)\\
}}|\nabla v|^{2} d x \leqslant \nu^{-1} \delta^{2} \int_{\substack{\Omega_{i}^{r} \\
i}} a_{i j} v_{x_{i}} v_{x_{j}} d x \\
& =\nu^{-1} \delta^{2} \lambda_{i}(r) \int_{\substack{\Omega^{r}\\
}} v^{2} d x=\nu^{-1} \delta^{2} \lambda_{L}(r) .
\end{aligned}
$$


Выберем $\delta$ так, чтобы $\nu^{-1} \delta^{2}{ }_{i}(r)=1 / 2$. Тогда

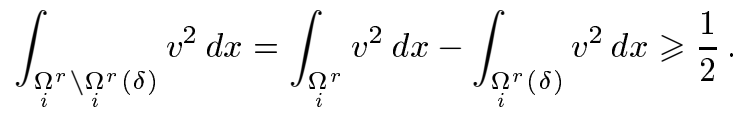

Отсюда

$$
\max _{\substack{\Omega_{i}^{r} \backslash \Omega_{i}^{r}(\delta) \\ v^{2}}} v^{2} \frac{1}{2 \operatorname{mes}\left(\Omega_{i}^{r}\right)} .
$$

Если предположить, что $\max v$ достигается на оси $O x_{1}$ в точке $y_{q}$, то будем иметь

$$
v\left(y_{q}\right) \geqslant \frac{1}{\sqrt{2 \operatorname{mes}\left(\Omega_{i}^{r}\right)}} .
$$

В общей ситуации, чтобы “дотянуться" от точки максимума $y^{*}$ до ближайшей точки $y_{q}$ на оси врашения, потребуется "боковая" цепочка касаюшихся шаров радиуса $\delta$ в некотором количестве $m$. Используя (64) и (62), устанавливаем, что

$$
m \leqslant \frac{f_{i}(r)}{\delta} \leqslant \gamma f_{i}(r) \underset{i}{\lambda^{1 / 2}}(r) \leqslant \gamma r \lambda_{i}^{1 / 2}(r) .
$$

Так что в любом случае

$$
v\left(y_{q}\right) \geqslant H^{-m} v\left(y^{*}\right) \geqslant \frac{H^{-m}}{\sqrt{2 \operatorname{mes}\left(\Omega_{i}^{r}\right)}} \geqslant \frac{a_{1}}{r^{n / 2}} H^{-m} .
$$

Итак, имеем

$$
\min _{B\left(\rho_{0}, z_{0}\right)} v \geqslant H^{-(q+m+1)} \frac{a_{1}}{r^{n / 2}} .
$$

Теперь нетрудно оценить число $c_{1}$ :

$$
c_{1} \geqslant H^{-(q+m+1)} \frac{a_{1}}{r^{n / 2}} \rho_{0}^{n} \omega_{1}=\frac{H^{-q-m} a_{2}}{r^{n / 2}},
$$

где $\omega_{1}$ - объем единичного шара в $R^{n}$.

Используя условия А) и В), оценим $q$. Имеем

$$
\begin{aligned}
q & =\underbrace{1+1+\cdots+1}_{q} \leqslant 1+Q \int_{y_{0}}^{y_{1}} \frac{\partial \rho}{f_{i}(\rho)}+\cdots+Q \int_{y_{q-2}}^{y_{q-1}} \frac{\partial \rho}{f_{i}(\rho)} \\
& \leqslant 1+Q \int_{1}^{r} \frac{\partial \rho}{f_{i}(\rho)} \leqslant b_{1} \frac{r}{f_{i}(r)} .
\end{aligned}
$$

Отсюда, применяя левое из неравенств (63), получаем

$$
m+q \leqslant \theta \underset{i}{\lambda_{i}^{1 / 2}}(r) .
$$

Используя последнее неравенство, из (65) благодаря (63) будем иметь при достаточно больших $r$

$$
\int_{\Omega_{i}^{r}} u_{i}^{2} d x \geqslant b_{2} H^{-\Theta r \lambda_{i}^{1 / 2}(r)} \exp \left(-2 \mu_{i}^{2} \lambda_{i}^{2}(r) t\right) .
$$

Полагая $r=r_{i}(t)$, в силу равенств (15) получим оценку снизу. 
4. Доказательство теоремы 3 основано на следуюшем утверждении.

ПРЕДЛОЖЕНИЕ 4. Пусть область $\Omega$ удовлетворяет условиям (23), (24) u (25). Пусть и $(t, x)$ - решение задачи (21), (22), (3). Тогда найдутся такое положительное число $\delta$, зависящее только от $n$, и такие числа $A, C$, зависящие от $n$ и $R_{0}\left(\operatorname{supp} \varphi \subset K_{R_{0}}\right)$, что для всех $t>0 u r^{6}>$ At справедливо неравенство

$$
\int_{\Omega_{r}^{\infty}} u^{2}(t, x) d x \leqslant C \exp \left(-\delta\left[\frac{r^{6}}{t}\right]^{1 / 5}\right)(1+t)\|\varphi\|_{L_{2}(\Omega)}^{2} .
$$

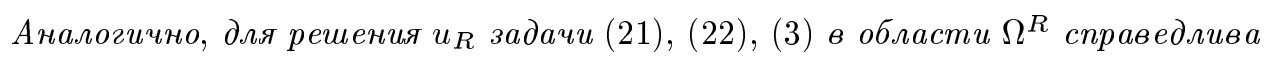
оценка вида (68):

$$
\int_{\Omega_{r}^{R}} u_{R}^{2}(t, x) d x \leqslant C \exp \left(-\delta\left[\frac{r^{6}}{t}\right]^{1 / 5}\right)(1+t)\|\varphi\|_{L_{2}(\Omega)}^{2} .
$$

ДокАЗАТЕльство. Так как доказательства оценок (68) и (69) проводятся аналогично, то мы рассмотрим здесь только доказательство оценки (68).

Для функции $p(t, x) \in L_{2}\left(D^{T}\right)$ обозначим через $p_{h}$ осреднение Стеклова

$$
p_{h}=\frac{1}{h} \int_{t}^{t+h} p(\tau, x) d \tau, \quad t+h<T .
$$

Пусть $v \in C^{3}\left(\overline{D^{T}}\right), v(t) \in C_{\Delta}^{3}(\bar{\Omega}) \forall t \geqslant 0,-$ произвольная пробная функция с ограниченньм носителем такая, что $v=0$ при $t \geqslant T-h$. Действуя как при доказательстве предложения 3 , устанавливаем равенство

$$
\int_{D^{T-h}}\left[\left(u_{h}\right)_{t} v+\nabla \Delta u_{h} \cdot \nabla \Delta v\right] d x d t=0 .
$$

Отметим, что равенство (70) справедливо и для любой функции $v \in H_{\Delta}^{0,3}\left(D^{T}\right)$ такой, что $v=0$ при $t \geqslant t_{1}, t_{1}<T-h$.

Пусть $s(r)$ - гладкая неотрицательная функция, равная нулю при $r \leqslant 0$ и единице при $r \geqslant 1$. Для области $\Omega$ существует функция $\xi(x)$ такая, что $\nabla \xi$ касается границы $\partial \Omega$ и $\xi\left(x_{1}, 0\right)=x_{1}$. А именно, можно взять $\xi(x)=g^{-1}\left(\left|x^{\prime}\right|^{2} / 2+g\left(x_{1}\right)\right)$, где $g^{\prime}\left(x_{1}\right)=f\left(x_{1}\right) / f^{\prime}\left(x_{1}\right), f\left(x_{1}\right)$ - функция из условия $(9)$, а $g^{-1}\left(x_{1}\right)$ - обратная функция к $g\left(x_{1}\right)$.

Введем обозначение $\widetilde{\Omega}_{a}^{b}=\{x \in \Omega: a<\xi(x)<b\}$. Рассмотрим функцию $\eta(x, r, \rho)=s((\xi-r) / \rho)$. Заметим, что $\left.\eta^{2} u_{h}\right|_{\partial \widetilde{\Omega}}=0$ и $\left.\Delta\left(\eta^{2} u_{h}\right)\right|_{\partial \widetilde{\Omega}}=\left[\eta^{2} \Delta u_{h}+\right.$ $\left.u_{h} \Delta \eta^{2}+2\left(\nabla \eta^{2} \cdot \nabla u_{h}\right)\right]\left.\right|_{\partial \widetilde{\Omega}}=0$, поскольку $\nabla \eta$ касается границы $\partial \Omega$. Подставив в (70) пробную функцию $v(t, x)=\left\{\begin{array}{ll}\eta^{2} u_{h}, & t<t_{1}, \\ 0, & t \geqslant t_{1},\end{array}\right.$ получим

$$
\int_{D^{t_{1}}}\left(\frac{1}{2}\left(\eta^{2} u_{h}^{2}\right)_{t}+\nabla \Delta\left(\eta^{2} u_{h}\right) \cdot \nabla \Delta u_{h}\right) d x d t=0 .
$$

Согласно предложению 2 решение задачи (21), (22), (3) принадлежит классу $C\left([0, \infty) \rightarrow L_{2}(\Omega)\right)$, следовательно,

$$
\int_{\Omega} u_{h}^{2}\left(t_{1}, x\right) \eta^{2} d x \rightarrow \int_{\Omega} u^{2}\left(t_{1}, x\right) \eta^{2} d x, \quad h \rightarrow 0 .
$$


Учитывая, что

$$
\int_{D^{t_{1}}}\left(\eta^{2} u_{h}\right)_{t} d x d t=\int_{\Omega}\left[u_{h}^{2}\left(t_{1}, x\right)-u_{h}^{2}(0, x)\right] \eta^{2} d x
$$

после предельного перехода в равенстве $(71)$ при $h \rightarrow 0$, прибавляя к обеим частям полученного равенства интеграл $\int_{D^{t} 1}(\nabla \Delta(\eta u))^{2} d x d t$, будем иметь

$$
\begin{gathered}
\frac{1}{2} \int_{\Omega}\left[u^{2}\left(t_{1}, x\right)-u^{2}(0, x)\right] \eta^{2} d x+\int_{D^{t_{1}}}(\nabla \Delta(\eta u))^{2} d x d t \\
=\int_{D^{t_{1}}}\left[(\nabla \Delta(\eta u))^{2}-\nabla \Delta u \cdot \nabla \Delta\left(\eta^{2} u\right)\right] d x d t
\end{gathered}
$$

Отметим, что $\nabla \eta=0$ при $\xi(x) \notin[r, r+\rho]$. Очевидны оценки

$$
|\nabla \eta| \leqslant \frac{c_{1}}{\rho}, \quad\left|\nabla^{2} \eta\right| \leqslant \frac{c_{2}}{\rho^{2}}, \quad|\nabla \Delta \eta| \leqslant \frac{c_{3}}{\rho^{3}}
$$

Здесь и далее $c_{i}$ и $c_{i}^{\prime}$ зависят от $n$ и области $\Omega$, точнее от функции $f$ в условии $(9)$. Последовательно разворачивая разность в правой части равенства (72), учитывая, что $\operatorname{supp} \varphi \subset K_{R_{0}}$, для всех $r>R_{0}, \rho>0$ получим

$$
\begin{aligned}
& \frac{1}{2} \int_{\widetilde{\Omega}_{r}^{\infty}} u^{2}\left(t_{1}, x\right) \eta^{2} d x+\int_{0}^{t_{1}} \int_{\widetilde{\Omega}_{r}^{\infty}}(\nabla \Delta(\eta u))^{2} d x d t \\
& \leqslant c_{5} \int_{0}^{t_{1}} \int_{\widetilde{\Omega}_{r}^{r+\rho}}\left[\left(\frac{\nabla u}{\rho^{2}}+\frac{u}{\rho^{3}}+\frac{\nabla^{2} u}{\rho}\right)^{2}+\left(\frac{\Delta u}{\rho}\right)^{2}\right. \\
& \left.\quad+\left|\left(\frac{\nabla u}{\rho^{2}}+\frac{u}{\rho^{3}}+\frac{\nabla^{2} u}{\rho}\right) \frac{\Delta u}{\rho}\right|+|\nabla \Delta u|\left(\frac{|\nabla u|}{\rho^{2}}+\frac{|u|}{\rho^{3}}\right)\right] d x d t .
\end{aligned}
$$

В книге [16; гл. I, $\S 1$, п. 5, формула (19)] О.А. Ладыженской установлено равенство

$$
\int_{\Omega}(\Delta v)^{2} d x=\int_{\Omega} \sum_{i, j=1}^{n} v_{x_{i} x_{j}}^{2} d x-\int_{S}\left(\frac{\partial v}{\partial n}\right)^{2} K d S
$$

для функций $v \in W_{2,0}^{2}(\Omega)$ с ограниченным носителем, причем $S$ - граница $\Omega$ дважды ограниченно-дифференцируема, где $K(x)$ - средняя кривизна поверхности $S$ в точке $x$. Если $K(x) \leqslant 0$ для всех $x \in S$, то нетрудно видеть, что

$$
\int_{\Omega} \sum_{i, j=1}^{n} v_{x_{i} x_{j}}^{2} d x \leqslant \int_{\Omega}(\Delta v)^{2} d x
$$

Предельным переходом неравенство (74) распространим на все функции из пространства $H_{\Delta}^{3}(\Omega)$. 
Положим для краткости записи $\eta_{r}=\eta(x, r, \rho), \eta_{r-\rho}=\eta(x, r-\rho, \rho)$. В силу выбора функции $\eta$ к функции $\eta_{r-\rho} u$ применимо неравенство $(74)$ в области $\widetilde{\Omega}_{r-\rho}^{\infty}$ :

$$
\int_{\tilde{\Omega}_{r}^{\infty}}\left|\nabla^{2} u\right|^{2} d x \leqslant \int_{\tilde{\Omega}_{r-\rho}^{\infty}}\left|\nabla^{2}\left(\eta_{r-\rho} u\right)\right|^{2} d x \leqslant \int_{\tilde{\Omega}_{r-\rho}^{\infty}}\left|\Delta\left(\eta_{r-\rho} u\right)\right|^{2} d x
$$

Используя неравенства (36), (37) и (75), применяя неравенство Коши и рассматривая такие $\varepsilon$ и $\rho$, что $\varepsilon \rho \geqslant 1$, неравенство (73) можно привести к виду

$$
\begin{aligned}
& \frac{1}{2} \int_{\tilde{\Omega}_{r}^{\infty}} u^{2}\left(t_{1}, x\right) \eta_{r}^{2} d x+\int_{0}^{t_{1}} \int_{\tilde{\Omega}_{r}^{\infty}}\left(\nabla \Delta\left(\eta_{r} u\right)\right)^{2} d x d t \\
& \quad \leqslant \frac{c_{6}}{\varepsilon \rho} \int_{0}^{t_{1}} \int_{\tilde{\Omega}_{r-\rho}^{\infty}}\left[\varepsilon^{6} \eta_{r-\rho}^{2} u^{2}+\left|\nabla \Delta\left(\eta_{r-\rho} u\right)\right|^{2}\right] d x d t
\end{aligned}
$$

Покажем, например, как оценивается слагаемое $\frac{1}{\rho^{2}} \int_{\widetilde{\Omega}_{r}}|\nabla u \cdot \nabla \Delta u| d x$. Применяя неравенство Коши, имеем

$$
\frac{1}{\rho^{2}} \int_{\widetilde{\Omega}_{r}}|\nabla u \cdot \nabla \Delta u| d x \leqslant \frac{1}{\rho^{2}}\left[\varepsilon_{1} \int_{\widetilde{\Omega}_{r}}|\nabla u|^{2} d x+\frac{1}{\varepsilon_{1}} \int_{\widetilde{\Omega}_{r}}|\nabla \Delta u|^{2} d x\right] .
$$

Теперь, применяя неравенство (37), устанавливаем соотношения

$$
\begin{aligned}
\frac{1}{\rho^{2}} \int_{\widetilde{\Omega}_{r}}|\nabla u \cdot \nabla \Delta u| d x \leqslant & \frac{1}{\rho^{2}}\left[\varepsilon_{1}\left(\varepsilon_{1} \int_{\widetilde{\Omega}_{r}} u^{2} d x+\frac{1}{4 \varepsilon_{1}^{2}} \int_{\widetilde{\Omega}_{r}}|\nabla \Delta u|^{2} d x\right)\right. \\
& \left.+\frac{1}{\varepsilon_{1}} \int_{\widetilde{\Omega}_{r}}|\nabla \Delta u|^{2} d x\right] .
\end{aligned}
$$

Положив $\varepsilon_{1}=\varepsilon^{2}$, из последнего выражения, учитьвая, что $\varepsilon \rho \geqslant 1$, легко получить

$$
\frac{1}{\rho^{2}} \int_{\widetilde{\Omega}_{r}}|\nabla u \cdot \nabla \Delta u| d x \leqslant \frac{c_{7}}{\varepsilon \rho}\left[\varepsilon^{6} \int_{\widetilde{\Omega}_{r}} u^{2} d x+\int_{\widetilde{\Omega}_{r}}|\nabla \Delta u|^{2} d x\right] .
$$

Введем обозначение

$$
H_{r}(t, \rho)=\int_{\tilde{\Omega}_{r}^{\infty}} u^{2}(t, x) \eta_{r}^{2} d x+\int_{0}^{t} \int_{\tilde{\Omega}_{r}^{\infty}}\left|\nabla \Delta\left(\eta_{r} u\right)\right|^{2} d x d t
$$

тогда из (76) последует

$$
H_{r}(t, \rho) \leqslant \frac{c_{8}}{\varepsilon \rho}\left[\varepsilon^{6} \int_{0}^{t} H_{r-\rho}(\tau, \rho) d \tau+H_{r-\rho}(t, \rho)\right]
$$

Докажем неравенство

$$
H_{r}(t, \rho) \leqslant A_{0}(1+t)
$$


$\forall r \geqslant R_{0}, \forall t \geqslant 0, \forall \rho \geqslant 1$, где $A_{0}=c^{\prime}\|\varphi\|^{2}$ - константа. Из неравенства (73) при $\rho \geqslant 1$ имеем

$$
H_{r}(t, \rho) \leqslant c_{9} \int_{0}^{t} \int_{\widetilde{\Omega}_{r}^{\infty}}\left[u^{2}+|\nabla u|^{2}+\left|\nabla^{2} u\right|^{2}+|\nabla \Delta u|^{2}\right] d x d t
$$

Применяя теперь $(74),(36)$ и (37), используя те же рассуждения, что и при выводе неравенства (76), получим

$$
H_{r}(t, \rho) \leqslant c_{10} \int_{0}^{t} \int_{\widetilde{\Omega}_{r}^{\infty}}\left[u^{2}+|\nabla \Delta u|^{2}\right] d x d t
$$

Требуемое неравенство (77) следует теперь из (34).

Аналогично (51) индукцией по $k$ устанавливается неравенство

$$
H_{r+k \rho}(t, \rho) \leqslant\left(\frac{2 c_{8}}{\rho}\right)^{k} t^{k / 6}\left[\frac{6^{k} 5 !}{(k+5) !}\right]^{1 / 6} A_{1}(1+t)
$$

$\forall r \geqslant R_{0}, \forall t \geqslant 0, \forall \rho \geqslant 1$.

Используя неравенство Стирлинга, из (78) несложно получить

$$
H_{r+k \rho}(t, \rho) \leqslant A_{3} \exp \left[-\frac{k}{6} \ln \left(\frac{k \rho^{6}}{c_{13} t}\right)\right](1+t) .
$$

Положим $\rho=\left(r-R_{0}\right) /(k+1)$, где $k$ - наибольшее натуральное число такое, что $\left(r-R_{0}\right)^{6}>2 c_{13}(k+1)^{5} e t, r \geqslant 2 R_{0}$. Сушествование такого натурального $k$ обеспечивается неравенством $r^{6}>A t$. Очевидно, $(k+2)^{5} \geqslant\left(r-R_{0}\right)^{6} /\left(2 c_{13} e t\right)$, откуда $k \geqslant\left(\left(r-R_{0}\right)^{6} /\left(2 c_{13} e t\right)\right)^{1 / 5}-2$. Подставляя эту оценку для $k$, из неравенства (79) имеем

$$
H_{r-\rho}(t, \rho)=H_{R_{0}+k \rho}(t, \rho) \leqslant A_{4} \exp \left(\frac{1}{3}-a_{1}\left[\frac{\left(r-R_{0}\right)^{6}}{t}\right]^{1 / 5}\right)(1+t) .
$$

Так как $\int_{\Omega_{r}^{\infty}} u^{2}(t, x) d x<H_{r-\rho}(t, \rho)$, то, учитывая, что $R_{0} \leqslant r / 2$, из (80) получим неравенство (68).

ДОКАЗАТЕЛЬСТво ТЕОРЕмЫ 3. Оценки (27) и (28) получаются единообразно с использованием предложения 4 , поэтому ограничимся доказательством оценки (27).

Рассмотрим вспомогательную функцию $\vartheta(r), r>0$, определенную в области $\Omega_{0}^{r}$ аналогично (56). Так как $\stackrel{\circ}{W} \underset{2}{1}(\Omega) \supset \stackrel{\circ}{W} \underset{2}{1}\left(\Omega_{0}^{r}\right)$, то $\vartheta(r) \leqslant \lambda(r)$, где $\lambda(r), r>0,-$ по-прежнему первое собственное число оператора Лапласа в области $\Omega_{0}^{r}$ с условием Дирихле на гранище. Теперь, используя (57) и (60), нетрудно установить, что функция $\vartheta(r)$ подчиняется неравенствам:

$$
c^{-1} \rho_{m}^{-2}(r) \leqslant \vartheta(r) \leqslant \lambda(r) \leqslant c \rho_{m}^{-2}(r), \quad c>1 .
$$


Отсюда следует, что для функций $u \in \stackrel{\circ}{W} \underset{2}{1}(\Omega)$ справедливо неравенство

$$
\int_{\Omega_{0}^{r}} u^{2} d x \leqslant c \rho_{m}^{2} \int_{\Omega_{0}^{r}}|\nabla u|^{2} d x
$$

Используя неравенства $(81),(37)$ и вложение $\widetilde{\Omega}_{0}^{r} \subset \Omega_{0}^{r}$, находим

$$
\begin{aligned}
& \frac{1}{c \rho_{m}^{2}}\left(\int_{\Omega} u^{2} d x-\int_{\widetilde{\Omega}_{r}^{\infty}} u^{2} d x\right)=\frac{1}{c \rho_{m}^{2}} \int_{\widetilde{\Omega}_{0}^{r}} u^{2} d x \leqslant \frac{1}{c \rho_{m}^{2}} \int_{\Omega_{0}^{r}} u^{2} d x \\
& \leqslant \int_{\Omega}|\nabla u|^{2} d x \leqslant \varepsilon \int_{\Omega} u^{2} d x+\frac{1}{4 \varepsilon^{2}} \int_{\Omega}|\nabla \Delta u|^{2} d x .
\end{aligned}
$$

Взяв $\varepsilon=1 /\left(2 c \rho_{m}^{2}\right)$, из последнего выражения получим

$$
\frac{1}{\left(c \rho_{m}^{2}\right)^{3}}\left(\frac{1}{2} \int_{\Omega} u^{2} d x-\int_{\widetilde{\Omega}_{r}^{\infty}} u^{2} d x\right) \leqslant \int_{\Omega}|\nabla \Delta u|^{2} d x
$$

Обозначим

$$
\beta=\max _{[0, T]} \int_{\widetilde{\Omega}_{r}^{\infty}} u^{2} d x
$$

Согласно предложению 2 функция $E(t)=\int_{\Omega} u^{2}(t, x) d x$ абсолютно непрерывна и

$$
\frac{d E}{d t}=-2 \int_{\Omega}|\nabla \Delta u|^{2} d x \leqslant \frac{1}{\left(c \rho_{m}^{2}\right)^{3}}(2 \beta-E(t)) .
$$

Поскольку $E(0)=\int_{\Omega} \varphi^{2} d x$, из последнего неравенства будем иметь

$$
E(t) \leqslant 2 \beta+\exp \left(-\frac{t}{\left(c \rho_{m}^{2}\right)^{3}}\right)\|\varphi\|^{2}
$$

Согласно предложению 4 из неравенства (82) нетрудно выявить, что

$$
\int_{\Omega} u^{2}(t, x) d x \leqslant M_{1}\left[\exp \left(-\varkappa_{1}\left[\frac{r^{6}}{t}\right]^{1 / 5}\right)+\exp \left(-\frac{t}{\left(c \rho_{m}^{2}\right)^{3}}\right)\right](1+t)\|\varphi\|_{L_{2}(\Omega)}^{2}
$$

Отметим также, что в силу условия (29) при больших значениях $t$ справедливо неравенство $r(t) \geqslant \max \left(2 R_{0}, r_{0}, \sqrt[6]{A t}\right)$. Используя равенства (26), подставляя в (83) $r=r(t)$, получим оценку $(27)$ теоремы 3 . 


\section{5. Оценка снизу для решения задачи (21), (22), (3).}

ДокАЗАТЕЛЬСТво ТЕОРЕмЫ 4 . Пусть функция $v(x)$ - первая нормированная в $L_{2}\left(\Omega^{r}\right)$ собственная функция в $\Omega^{r}$ :

$$
\Delta v=-\lambda(r) v,\left.\quad v\right|_{\partial \Omega^{r}}=0 .
$$

Если $B\left(\rho_{m}, z\right) \subset \Omega^{r}$ - максимальный шар в $\Omega^{r}$, то, как известно,

$$
\frac{1}{c \rho_{m}^{2}} \leqslant \lambda\left(\Omega^{r}\right) \leqslant \lambda\left(B\left(\rho_{m}, z\right)\right) \leqslant \frac{c}{\rho_{m}^{2}}, \quad c>1 .
$$

Выберем произвольную точку $z_{0} \in \Omega$ на луче $O x_{1}$ такую, что $\left|z_{0}\right|>P$. Рассмотрим решение задачи $(21),(22),(3)$ в $\Omega^{r}$ с начальной функцией $\chi_{B\left(\rho_{0}, z_{0}\right)}$, где $B\left(\rho_{0}, z_{0}\right)$ - допустимый шар. Оно представляется в виде

$$
u_{r}(t, x)=\sum_{j=1}^{\infty} c_{j} \exp \left(-\lambda_{j}(r) t\right) v_{j}(x)
$$

где $v_{j}(x)$ - собственные функции оператора $\Delta^{3}$ в области $\Omega^{r}$. В нашем случае собственная функция $v_{1}$ оператора $\Delta^{3}$ одновременно является первой собственной функцией оператора $\Delta$, причем $\lambda_{1}(r)=\lambda^{3}(r) \leqslant c^{3} / \rho_{m}^{6}(r)$. Имеем

$$
\int_{\substack{\Omega^{r} \\ r}} u_{r}^{2} d x \geqslant c_{1}^{2} \exp \left(-2 \lambda_{1}(r) t\right) \geqslant c_{1}^{2} \exp \left(-\frac{2 c^{3}}{\rho_{m}^{6}(r)} t\right),
$$

где $c_{1}=\int_{\substack{\Omega^{r} \\ \text { 促 }}} \chi_{B\left(\rho_{0}, z_{0}\right)} v_{1} d x$. Число $c_{1}$ оценено в неравенствах $(66)$ и $(67)$. Используя последнее, (60) и (63), будем иметь

$$
\int_{\Omega^{r}} u_{r}^{2} d x \geqslant H^{-\frac{2 \theta r}{\rho_{m}(r)}} \frac{a_{2}^{2}}{r^{n / 2}} \exp \left(-\frac{2 c^{3}}{\rho_{m}^{6}(r)} t\right) \geqslant b_{2} H^{-\frac{3 \theta r}{\rho_{m}(r)}} \exp \left(-\frac{2 c^{3}}{\rho_{m}^{6}(r)} t\right) .
$$

Пока $r$ было произвольным (достаточно большим). Теперь положим $r=r(t)$ и в силу равенств (26) получим оценку снизу.

\section{Список литературы}

1. Гущин $A$. K. Об оценках решений краевых задач для параболических уравнений второго порядка // Труды МИАН. 1973. Т. 126. С. 5-45.

2. Гущин $A$. K. Стабилизация решений второй краевой задачи для параболического уравнения // Матем. сб. 1976. Т. 101 (143). № 4. С. 459-499.

3. Гущин A.K. О равномерной стабилизации решений второй смешанной задачи для параболического уравнения // Матем. сб. 1982. Т. 119 (161). № 4. С. 451-508.

4. Гущин A.K., Михайлов В. П. О стабилизации решения задачи Коши для одномерного параболического уравнения // Труды МИАН. 1971. Т. 112. С. 181-202.

5. Ушаков В.И. Стабилизация решений третьей смешанной задачи для параболического уравнения второго порядка в нецилиндрической области // Матем. сб. 1980. Т. 111 (153). № 1. C. 95-115.

6. Гущин А.К., Михайлов В. П., Михайлов Ю.А. О равномерной стабилизации решения второй смешанной задачи для параболического уравнения второго порядка // Матем. сб. 1985. Т. 128(170). С. 147-168. 
7. Богоявленский О.В., Владимиров В.С., Волович И.В., Гущин А.К., Дрожжинов Ю. Н., Жаринов В. В., Михайлов В. П. Краевые задачи математической физики // Труды МИАН. 1986. Т. 175. С. 63-102.

8. Тедеев $A . \Phi$. Стабилизация решений первой смешанной задачи для квазилинейного параболического уравнения высокого порядка // Дифференц. уравнения. 1989. Т. 25. № 3 . C. $491-498$.

9. Мукминов $\Phi . X$. Об убывании нормы решения смешанной задачи для параболического уравнения высокого порядка // Дифференц. уравнения. 1987. Т. 23. № 10. С. 1172-1780.

10. Мукминов $\Phi . X$. Стабилизация решений первой смешанной задачи для параболического уравнения второго порядка // Матем. сб. 1980. Т. 111 (153). № 4. С. 503-521.

11. Вишик М. И. Смешанные краевые задачи и приближенный метод их решения // Докл. AH CCCP. 1954. Т. 97. № 2. С. 193-196.

12. Берс Л., Джсон Ф., Шехтер М. Уравнения с частными производньми. М.: Мир, 1966.

13. Гущин $A . K$. Некоторые свойства обобщенного решения второй краевой задачи для параболического уравнения // Матем. сб. 1975. Т. 97 (139). С. 242-261.

14. Мукминов $\Phi . X$. Стабилизация решений первой смешанной задачи для системы уравнений Навье-Стокса // Дис. . . д докт. физ.-матем. наук. М.: МИРАН, 1994.

15. Кожсевникова Л. М., Мукминов $\Phi$. Х. Оценки скорости стабилизации при $t \rightarrow \infty$ решений первой смешанной задачи для квазилинейной системы параболических уравнений второго порядка // Матем. сб. 2000. Т. 191. № 2. С. 91-131.

16. Лады жсенская О. А. Математические вопросы динамики вязкой несжимаемой жидкости. М.: Физматгиз, 1961.

Стерлитамакский государственный

Поступила в редакцию

педагогический институт

21.07.2003

E-mail: mfkh@mail.rb.ru

radosti@mail.rb.ru 